\title{
Sampling Sets for the Nevanlinna class
}

\section{Xavier Massaneda and Pascal J. Thomas}

\begin{abstract}
We propose a definition of sampling set for the Nevanlinna and Smirnov classes in the disk and show its equivalence with the notion of determination set for the same classes. We also show the relationship with determination sets for related classes of functions and deduce a characterization of Smirnov sampling sets. For Nevanlinna sampling we give general conditions (necessary or sufficient), from which we obtain precise geometric descriptions in several regular cases.
\end{abstract}

\section{Introduction}

Let $\Lambda$ be a subset in the unit disk $\mathbb{D}$. In general $\Lambda$ is called sampling for a space of holomorphic functions $X$ when any function $f \in X$ is determined by its restriction $f \mid \Lambda$, with control of norms. For Banach spaces $X$ it is usually clear what that control of norms means, but for the spaces we have in mind the situation is not so obvious. Consider the Nevanlinna class

$$
\mathcal{N}=\left\{f \in \operatorname{Hol}(\mathbb{D}): \lim _{r \rightarrow 1} \frac{1}{2 \pi} \int_{0}^{2 \pi} \log ^{+}\left|f\left(r e^{i \theta}\right)\right| d \theta<\infty\right\},
$$

which is not a Banach space, but enjoys the structure of complete metric space with the distance $d(f, g)=N(f-g)$ induced by

$$
N(f)=\lim _{r \rightarrow 1} \frac{1}{2 \pi} \int_{0}^{2 \pi} \log \left(1+\left|f\left(r e^{i \theta}\right)\right|\right) d \theta .
$$

The subharmonicity of $\log (1+|f|)$ yields the pointwise estimate

$$
(1-|z|) \log (1+|f(z)|) \leq 2 N(f),
$$

which shows that convergence in the distance $d$ implies uniform convergence on compact sets [11, Proposition 1.1].

2000 Mathematics Subject Classification: 30D50, 30C80, 30E05.

Keywords: Sampling sets, determination sets, Nevanlinna class, Smirnov class. 
The Nevanlinna class $\mathcal{N}$ coincides with the set of functions $f \in \operatorname{Hol}(\mathbb{D})$ such that $\log (1+|f|)$ admits a harmonic majorant (see [4, p.69] or (2.2) later on). The value $N(f)$ can then be rewritten in terms of an extremal problem for harmonic majorants. Let $\operatorname{Har}_{+}(\mathbb{D})$ denote the space of non-negative harmonic functions in the disk; then

$$
N(f)=\inf \left\{h(0): h \in \operatorname{Har}_{+}(\mathbb{D}) \text { with } \log (1+|f|) \leq h\right\}
$$

This expression makes sense for any $f$ measurable in $\mathbb{D}$, in particular for a restriction $f \mid \Lambda$ extended to be 0 on $\mathbb{D} \backslash \Lambda$, and suggests the following definition.

Definition. A set $\Lambda$ is sampling for $\mathcal{N}$ if there exists $C>0$ such that

$$
N(f) \leq N(f \mid \Lambda)+C \quad \forall f \in \mathcal{N} .
$$

In Section 2 we study first the relationship between Nevanlinna sampling sets and determination sets for the same class and for the space $\mathcal{H}^{\infty}$ of bounded holomorphic functions. We prove that sampling and determination sets for $\mathcal{N}$ are the same. Also, from the characterization of $\mathcal{H}^{\infty}$ determination sets given by Brown, Shields and Zeller [2] we deduce a complete description of sampling sets for the Smirnov class

$\mathcal{N}^{+}=\left\{f \in \mathcal{N}: \lim _{r \rightarrow 1} \int_{0}^{2 \pi} \log ^{+}\left|f\left(r e^{i \theta}\right)\right| \frac{d \theta}{2 \pi}=\frac{1}{2 \pi} \int_{0}^{2 \pi} \log ^{+}\left|f^{*}\left(e^{i \theta}\right)\right| d \theta<\infty\right\}$.

Here $f^{*}\left(e^{i \theta}\right)$ denotes the non-tangential limit of $f$ at the boundary point $e^{i \theta}$.

Next we study the relationship between sampling sets for $\mathcal{N}$ and determination sets for the class $\operatorname{Har}_{ \pm}(\mathbb{D})$ of harmonic functions which can be written as the difference of two positive harmonic functions. This is relevant because the Riesz-Smirnov factorization implies that for any $f \in \mathcal{N}$ there exist a Blaschke product $B$ and $h \in \operatorname{Har}_{ \pm}(\mathbb{D})$ such that $\log |f|=\log |B|+h$. From the characterization of determination sets for $\operatorname{Har}_{ \pm}(\mathbb{D})$ given by Hayman and Lyons [5] we deduce a necessary geometric condition for sampling in $\mathcal{N}$. Some examples show that this condition is far from being sufficient.

In Section 3 we give general conditions for Nevanlinna sampling (Theorem 3.2), which in Section 4 are used to obtain a precise geometric description for three different types of regular sampling sets: fine nets of points, regular sequences on circles tending to the unit circle, and uniformly dense unions of hyperbolic disks, as considered by Ortega-Cerdà and Seip in [8] .

A final remark about notation. The expression $A \preceq B$ means that there exists a constant $C>0$, independent of whatever arguments are involved, such that $A \leq C B$. If both $A \preceq B$ and $B \preceq A$ then we write $A \simeq B$. 


\section{Determination sets and necessary conditions}

In this section we describe the relationship between our definition of sampling and other related notions studied previously.

\subsection{Sampling and determination sets}

We begin with an easy observation: in the definition of sampling given in the introduction $N(f)$ can be equivalently replaced by

$$
\begin{aligned}
N_{+}(f) & =\lim _{r \rightarrow 1} \frac{1}{2 \pi} \int_{0}^{2 \pi} \log ^{+}\left|f\left(r e^{i \theta}\right)\right| d \theta \\
& =\inf \left\{h(0): h \in \operatorname{Har}_{+}(\mathbb{D}) \text { with } \log ^{+}|f| \leq h\right\} .
\end{aligned}
$$

This is an immediate consequence of the inequalities

$$
\log ^{+}|f| \leq \log (1+|f|) \leq \log 2+\log ^{+}|f| .
$$

Also, if we use $N_{+}(f)$ instead of $N(f)$ the constant $C$ can be assumed to be 0 , as the following Lemma shows.

Lemma 2.1. A set $\Lambda$ is sampling for $\mathcal{N}$ if and only if $N_{+}(f)=N_{+}(f \mid \Lambda)$ for all $f \in \mathcal{N}$.

Proof. Of course, we only need to see that the equality is necessary. Since $\mathcal{N}$ is an algebra, the sampling inequality $N_{+}(f) \leq N_{+}(f \mid \Lambda)+C$ yields automatically

$$
N_{+}\left(f^{n}\right) \leq N_{+}\left(f^{n} \mid \Lambda\right)+C \quad \forall f \in \mathcal{N} \quad \forall n \in \mathbb{N} .
$$

By definition $N_{+}\left(f^{n}\right)=n N_{+}(f)$, so

$$
N_{+}(f) \leq N_{+}(f \mid \Lambda)+\frac{C}{n} \quad \forall f \in \mathcal{N} \quad \forall n \in \mathbb{N},
$$

and the result follows letting $n$ tend to $\infty$.

Let us consider also two related notions for a set $\Lambda$ in $\mathbb{D}$. A priori, one seems weaker and the other stronger than the sampling property.

Definition. A set $\Lambda$ is a determination set for $\mathcal{N}$ if $\mathcal{N} \cap L^{\infty}(\Lambda) \subset \mathcal{H}^{\infty}$, i.e. if any $f \in \mathcal{N}$ with $\sup _{\Lambda}|f|<\infty$ must be bounded on the whole unit disk.

A set $\Lambda$ is strongly sampling for $\mathcal{N}$ if whenever $f \in \mathcal{N}$ and $h \in \operatorname{Har}_{+}(\mathbb{D})$ are such that $\log ^{+}|f(\lambda)| \leq h(\lambda)$ for all $\lambda \in \Lambda$, then necessarily $\log ^{+}|f| \leq h$.

When $\Lambda$ is strongly sampling the distance $d(f, g)$ between two functions $f, g \in \mathcal{N}$ coincides with the distance between their restrictions $f \mid \Lambda$ and $g \mid \Lambda$. 
Remark 1. A set $\Lambda$ is a determination set for $\mathcal{H}^{\infty}$ when $\|f\|_{\infty}=\sup _{\Lambda}|f|$ for all $f \in \mathcal{H}^{\infty}$. It is easy to see that determination sets for $\mathcal{N}$ are also determination sets for $\mathcal{H}^{\infty}$, which therefore satisfy $\|f\|_{\infty}=\sup _{\Lambda}|f|$ for all $f \in \mathcal{N}$.

Indeed, assume that there exists $f \in \mathcal{H}^{\infty}$ such that $\|f\|_{\infty}=1$ and $\sup _{\Lambda}|f|=s<1$. Take $\left\{z_{k}\right\}_{k} \subset \mathbb{D}$ such that $\lim _{k}\left|f\left(z_{k}\right)\right|=1$ and consider any accumulation point $\zeta \in \mathbb{T}$ of $\left\{f\left(z_{k}\right)\right\}_{k}$. Then the function $g=1 /(\zeta-f)$ belongs to $\mathcal{N}$, is not bounded, but $\sup _{\Lambda}|g| \leq 1 /(1-s)$.

Brown, Shields and Zeller showed that $\Lambda$ is a determination set for $\mathcal{H}^{\infty}$ if and only if the set $N T(\Lambda)$ consisting of the $\zeta \in \mathbb{T}$ which are a non-tangential limit of points in $\Lambda$ has full measure, i.e. $|N T(\Lambda)|=2 \pi$ [2]. It was shown in [12] that the same condition also characterizes sampling sets for the Hardy spaces $\mathcal{H}^{p}(0<p<\infty)$, if appropriately defined. This condition is therefore necessary for $\Lambda$ to be a determination set for $\mathcal{N}$.

Our first result shows that the previous notions are all equivalent.

Theorem 2.2. Let $\Lambda$ be a subset of $\mathbb{D}$. The following properties are equivalent:

(a) $\Lambda$ is a sampling set for $\mathcal{N}$.

(b) $\Lambda$ is a determination set for $\mathcal{N}$.

(c) $\Lambda$ is a strongly sampling set for $\mathcal{N}$.

It is clear from (c) that the sampling property is invariant under automorphisms of the disk: if $\Lambda$ is sampling for $\mathcal{N}$ and $\phi(z)=e^{i \theta} \frac{z-a}{1-\bar{a} z}, a \in \mathbb{D}$, is an automorphism of $\mathbb{D}$, then $\phi(\Lambda)$ is also sampling for $\mathcal{N}$.

Before the proof we need to recall some well-known facts about the structure of the Nevanlinna class (general references are e.g. [4], [7] or [9]).

For a set $Z \subset \mathbb{D}$ with multiplicities, the Blaschke product with zeros on $Z$ is

$$
B_{Z}(z):=\prod_{a \in Z} \frac{\bar{a}}{|a|} \frac{a-z}{1-z \bar{a}}
$$

where the points are repeated according to multiplicities. This is convergent, not identically equal to 0 , if and only if $\sum_{a \in Z}(1-|a|)<\infty$. When this is the case, we say that $Z$ is a Blaschke sequence, or verifies the Blaschke condition.

A function $f$ is called outer if it can be written in the form

$$
f(z)=C \exp \left\{\int_{0}^{2 \pi} \frac{e^{i \theta}+z}{e^{i \theta}-z} \log v\left(e^{i \theta}\right) \frac{d \theta}{2 \pi}\right\},
$$

where $|C|=1, v>0$ a.e. on $\mathbb{T}$ and $\log v \in L^{1}(\mathbb{T})$. Such a function is the quotient $f=f_{1} / f_{2}$ of two bounded outer functions $f_{1}, f_{2} \in \mathcal{H}^{\infty}$ with 
$\left\|f_{i}\right\|_{\infty} \leq 1, i=1,2$. In particular, the weight $v$ is given by the boundary values of $\left|f_{1} / f_{2}\right|$. Setting $w=\log v$, we have

$$
\log |f(z)|=P[w](z):=\int_{0}^{2 \pi} P_{z}\left(e^{i \theta}\right) w\left(e^{i \theta}\right) \frac{d \theta}{2 \pi},
$$

where

$$
P_{z}\left(e^{i \theta}\right):=\frac{1-|z|^{2}}{\left|e^{i \theta}-z\right|^{2}}
$$

denotes the Poisson kernel at $z \in \mathbb{D}$.

In general, for any finite measure $\mu$ on $\mathbb{T}$, the Poisson integral of $\mu$ is the harmonic function given by

$$
P[\mu](z):=\int_{0}^{2 \pi} P_{z}\left(e^{i \theta}\right) d \mu(\theta) .
$$

Another important family in this context are inner functions: $I \in \mathcal{H}^{\infty}$ such that $|I|=1$ almost everywhere on $\mathbb{T}$. Any inner function $I$ can be factorized into a Blaschke product $B$ carrying the zeros of $I$, and a singular inner function $S$ defined by

$$
S(z)=\exp \left\{-\int_{0}^{2 \pi} \frac{e^{i \theta}+z}{e^{i \theta}-z} d \mu\left(e^{i \theta}\right)\right\},
$$

for some positive Borel measure $\mu$ singular with respect to Lebesgue measure.

According to the Riesz-Smirnov factorization, any function $f \in \mathcal{N}$ is represented as

$$
f=\alpha \frac{B S_{1} f_{1}}{S_{2} f_{2}}
$$

with $f_{i}$ outer, $\left\|f_{i}\right\|_{\infty} \leq 1, S_{i}$ singular inner, $B$ a Blaschke product and $|\alpha|=1$.

Remark 2. Let $\operatorname{Har}_{ \pm}(\mathbb{D})$ denote the set of harmonic functions $h$ that can be written $h=h_{1}-h_{2}$, with $h_{1}, h_{2} \in \operatorname{Har}_{+}(\mathbb{D})$. The factorization above shows that for $f \in \mathcal{N}$ there exist always $h \in \operatorname{Har}_{ \pm}(\mathbb{D})$ and a Blaschke product $B$ such that

$$
\log |f|=\log |B|+h
$$

and reciprocally, for any $h \in \operatorname{Har}_{ \pm}(\mathbb{D})$ and any Blaschke product $B$ there exists $f \in \mathcal{N}$ satisfying (2.2). 
Notice also that when $\Lambda$ is a determination set for $\mathcal{N}$ and $f, g \in \mathcal{N}$ are such that $|f(\lambda)| \leq|g(\lambda)|$ for all $\lambda \in \Lambda$, then $|B f| \leq|g|$, where $B$ indicates the Blaschke product associated to the zeros of $g$. To see this factorize $g=B g_{0}$, with $g_{0}$ non-vanishing. Then $|f(\lambda)| /\left|g_{0}(\lambda)\right| \leq|B(\lambda)| \leq 1$ and by hypothesis $|f| \leq\left|g_{0}\right|$, as desired.

Proof of Theorem 2.2. (c) $\Rightarrow$ (a) is immediate from the definition.

(a) $\Rightarrow\left(\right.$ b). Let $f \in \mathcal{N}$ with $s_{\Lambda}=: \sup _{\Lambda}|f|<\infty$ and consider $g=f / s_{\Lambda} \in \mathcal{N}$. Since $\Lambda$ is sampling and $\log ^{+}|g(\lambda)|=0$ for all $\lambda \in \Lambda$ we have, according to Lemma 2.1, $N_{+}(g)=N_{+}(g \mid \Lambda)=0$. Thus $\int_{0}^{2 \pi} \log ^{+}\left|g\left(r e^{i \theta}\right)\right| d \theta=0$ for all $r<1$, hence $\|g\|_{\infty} \leq 1$.

$(\mathrm{b}) \Rightarrow(\mathrm{c})$. Let $f \in \mathcal{N}$ and $h \in \operatorname{Har}_{+}(\mathbb{D})$ be such that $\log |f(\lambda)| \leq h(\lambda)$ for all $\lambda \in \Lambda$. By Remark 2, there exists a function $g \in \mathcal{N}$ such that $\log |g|=\log |f|-h$. We have then $\log |g(\lambda)| \leq 0$ for all $\lambda \in \Lambda$, and as pointed out in Remark 1, this implies $\|g\|_{\infty} \leq 1$, i.e. $\log |g|=\log |f|-h \leq 0$.

\subsection{Sampling in the Smirnov class}

All the definitions and proofs above can be similarly given for the Smirnov class $\mathcal{N}^{+}$defined in the introduction. The Smirnov class consists of those $f \in \mathcal{N}$ for which the harmonic majorant of $\log ^{+}|f|$ is quasi-bounded (the Poisson integral of some $w \in L^{1}(\mathbb{T})$ ). Equivalently, it consists of those $f \in \mathcal{N}$ with no singular factor $S_{2}$ in the factorization (2.1).

The geometric description of sampling sequences for $\mathcal{N}^{+}$is a straightforward consequence of the results in [2] and Remark 1. Recall that $N T(\Lambda)$ denotes the non-tangential accumulation set of $\Lambda$ in $\mathbb{T}$.

Theorem 2.3. Let $\Lambda$ be a subset of $\mathbb{D}$. The following properties are equivalent:

(a) $\Lambda$ is a sampling set for $\mathcal{N}^{+}$.

(b) $\Lambda$ is a determination set for $\mathcal{N}^{+}$.

(c) $\Lambda$ is a strongly sampling set for $\mathcal{N}^{+}$.

(d) $|N T(\Lambda)|=2 \pi$.

Proof. The equivalence between (a), (b) and (c) is seen as in Theorem 2.2.

The necessity of $(d)$ is pointed out in Remark 1 . The sufficency is immediate: for almost every $\theta \in[0,2 \pi)$ there exists a sequence $\left\{\lambda_{k}\right\}_{k} \subset \Lambda$ tending non-tangentially to $e^{i \theta}$, and therefore $f^{*}\left(e^{i \theta}\right)=\lim _{k \rightarrow \infty} f\left(\lambda_{k}\right)[4$, Theorem 5.3]. Then, if $f \in \mathcal{N}^{+}$and $h \in \operatorname{Har}_{+}(\mathbb{D})$ are such that $\log ^{+}|f(\lambda)| \leq h(\lambda)$ for all $\lambda \in \Lambda$ we have $\log ^{+}\left|f^{*}\left(e^{i \theta}\right)\right| \leq h\left(e^{i \theta}\right)$ a.e. $\theta \in \mathbb{T}$. This yields $N_{+}(f) \leq N_{+}(f \mid \Lambda)$. 


\subsection{Determination sets for harmonic functions and a necessary condition for Nevanlinna sampling}

From previous results on determination sets for harmonic functions and the equivalences of Theorem 2.2 we deduce a first necessary condition for Nevanlinna sampling (Corollary 2.4). This can be obtained directly, as shown in the Appendix.

Given $z, w \in \mathbb{D}$ let

$$
\rho(z, w):=\left|\frac{z-w}{1-z \bar{w}}\right|
$$

stand for the the pseudohyperbolic distance. For $r \in(0,1)$ and $z \in \mathbb{D}$ let $D(z, r)=\{w \in \mathbb{D}: \rho(z, w)<r\}$.

A sequence $\Lambda=\left\{\lambda_{k}\right\}_{k}$ is called separated when

$$
\inf _{j \neq k} \rho\left(\lambda_{j}, \lambda_{k}\right)>0
$$

For any set $\Lambda \subset \mathbb{D}$ and $\delta \in(0,1)$, consider the pseudohyperbolic dilation

$$
\Lambda^{\delta}=\bigcup_{\lambda \in \Lambda} D(\lambda, \delta)
$$

and given $\zeta \in \mathbb{T}$ denote

$$
I(\Lambda, \zeta, \delta):=\int_{\Lambda^{\delta}} \frac{1}{|\zeta-z|^{2}} d m(z)
$$

where $d m$ stands for the usual area measure.

We note that for any fixed $\zeta \in \mathbb{T}$, the values $I(\Lambda, \zeta, \delta)$ are either finite or infinite simultaneously for all values of $\delta \in(0,1)$. That they are finite is equivalent to the fact that for any maximal separated subsequence $\Lambda^{\prime} \subset \Lambda$, we have

$$
\sum_{\lambda \in \Lambda^{\prime}}\left(\frac{1-|\lambda|^{2}}{|\zeta-\lambda|}\right)^{2}=\sum_{\lambda \in \Lambda^{\prime}}\left(1-|\lambda|^{2}\right) P_{\lambda}(\zeta)<\infty
$$

We recall the following characterization of determination sets for the class $\operatorname{Har}_{ \pm}(\mathbb{D})$ given by Hayman and Lyons [5]. This is elaborated upon in [3].

Theorem A. Let $\Lambda \subset \mathbb{D}$. The following properties are equivalent.

(a) $\sup _{\Lambda} h=\sup _{\mathbb{D}} h$ for all $h \in \operatorname{Har}_{ \pm}(\mathbb{D})$.

(b) There exists $\delta \in(0,1)$ such that $I(\Lambda, \zeta, \delta)=\infty$ for all $\zeta \in \mathbb{T}$.

We shall call the sets satisfying these conditions Hayman-Lyons sets. 
Condition (b) is more restrictive than Brown, Shields and Zeller's condition $|N T(\Lambda)|=2 \pi$. In fact, $|N T(\Lambda)|=2 \pi$ is equivalent to $I(\Lambda, \zeta, \delta)=\infty$ a.e. $\zeta \in \mathbb{T}[3$, Corollary 2]. On the other hand, it is clear that if $N T(\Lambda)=\mathbb{T}$ then (b) is satisfied, since the Poisson kernel $P(z, \zeta)$ is bounded below by $C\left(1-|\lambda|^{-1}\right)$ in any Stolz angle with vertex at $\zeta$.

Corollary 2.4. A Nevanlinna sampling set is a Hayman-Lyons set.

Proof. By Theorem 2.2, $\Lambda$ is a determination set for $\mathcal{N}$, hence $\sup _{\Lambda} \log |f|=$ $\sup _{\mathbb{D}} \log |f|$ for all $f \in \mathcal{N}$. By Remark 2, this implies (a) in Theorem A.

The Hayman-Lyons condition is not sufficient for sampling in $\mathcal{N}$, as shown in the following example.

Example 1. Take a dyadic partition of the disk: for any $(n, k)$ in the set of indices $\mathcal{I}=\left\{(n, k): n \in \mathbb{N}, 0 \leq k \leq 2^{n}-1\right\}$ consider the interval

$$
I_{n, k}:=\left\{e^{i \theta}: \theta \in\left[2 \pi k 2^{-n}, 2 \pi(k+1) 2^{-n}\right)\right\},
$$

and the associated Whitney partition in "dyadic squares":

$$
Q_{n, k}:=\left\{r e^{i \theta}: e^{i \theta} \in I_{n, k}, 1-2^{-n} \leq r<1-2^{-n-1}\right\} .
$$

Observe that the pseudohyperbolic diameter of each Whitney square $Q_{n, k}$ is bounded between two absolute constants.

Let $\Lambda$ be the sequence consisting of the centers $c_{n, k}$ of $Q_{n, k}$. An immediate computation shows that $I(\Lambda, \zeta, \delta) \simeq \int_{\mathbb{D}} \frac{d m(z)}{|z-\zeta|^{2}}=\infty$ for every $\zeta \in \mathbb{T}$, or equivalently:

$$
\begin{aligned}
\sum_{n=1}^{\infty} \sum_{k=0}^{2^{n}-1}\left(1-\left|c_{n, k}\right|^{2}\right) P_{c_{n, k}}(\zeta) & =\sum_{n=1}^{\infty} \sum_{k=0}^{2^{n}-1}\left(\frac{1-\left|c_{n, k}\right|^{2}}{\left|\zeta-c_{n, k}\right|}\right)^{2} \\
& \simeq \sum_{n=1}^{\infty} \sum_{k=0}^{2^{n}-1}\left(\frac{2^{-n}}{2^{-n}+k 2^{-n}}\right)^{2}=\infty
\end{aligned}
$$

Therefore $\Lambda$ is a Hayman-Lyons set.

In order to see that $\Lambda$ is not a determination set for $\mathcal{N}$ fix $\zeta=1 \in \mathbb{T}$ and consider a horocycle $\left\{z: P_{z}(1)=c\right\}$, the boundary of the euclidian disk $B\left(\frac{c}{1+c}, \frac{1}{1+c}\right)$ (see Figure 1$)$. Then $Z=\Lambda \cap B\left(\frac{c}{1+c}, \frac{1}{1+c}\right)$ is a Blaschke sequence:

$$
\sum_{a \in Z} 1-|a| \simeq \sum_{n=1}^{\infty} \sum_{0 \leq k<\sqrt{2^{n}}}\left(1-\left|c_{n, k}\right|\right) \simeq \sum_{n=1}^{\infty} 2^{-n / 2}<\infty .
$$




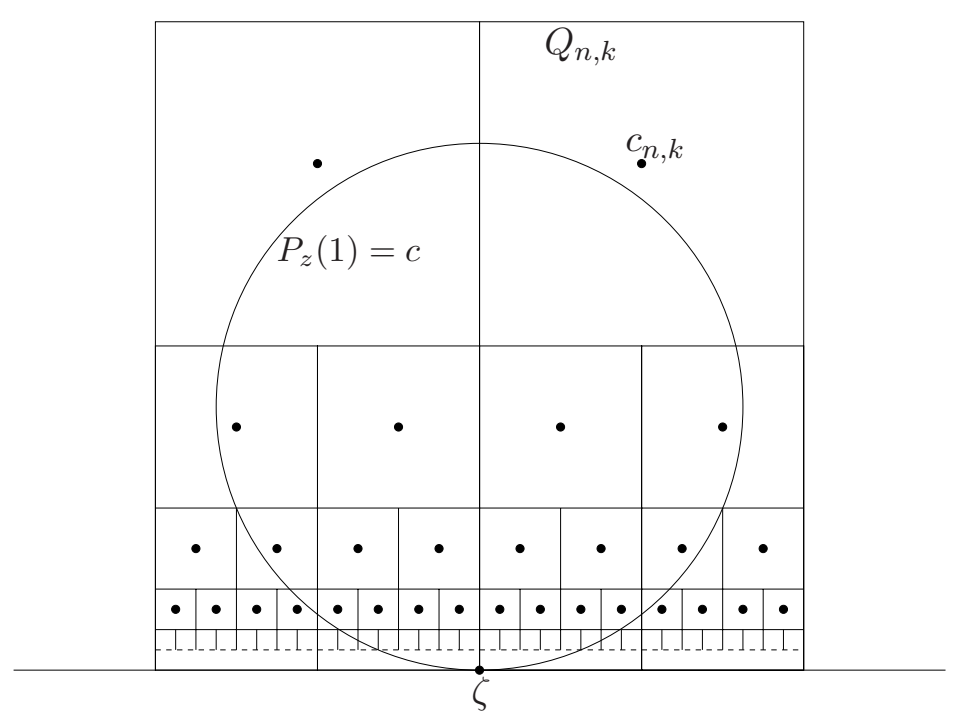

Figure 1. Upper half-plane representation of $\Lambda$ and the horocycle $P_{z}(1)=c$.

Therefore, there exists $f \in \mathcal{N}$ such that

$$
\log |f(z)|=\log \left|B_{Z}(z)\right|+P_{z}(1) \quad z \in \mathbb{D} .
$$

Clearly $\log |f(\lambda)| \leq c$ for all $\lambda \in \Lambda$. The fact that $f$ is not bounded rests on the following more general lemma.

Lemma 2.5. [11, Lemma 2.3] For any Blaschke product $B$ and any $\zeta \in \mathbb{T}$,

$$
\limsup _{r \rightarrow 1}(1-r) \log |B(r \zeta)|=0 .
$$

This implies that $\lim \sup _{r \rightarrow 1}(1-r) \log |f(r)|=\lim \sup _{r \rightarrow 1}(1-r) P_{r}(1)=2$, so $f$ cannot be bounded on $(0,1)$.

We finish this section by proving a general necessary condition in terms of approach regions.

Let

$$
\begin{aligned}
\mathcal{F}=\left\{\psi:[0,1) \longrightarrow \mathbb{R}_{+}\right. \text {non-decreasing, continuous, } \\
\text { with } \left.\psi(0)=0 \text { and } \int_{0} \psi(x) / x^{2} d x<\infty\right\} .
\end{aligned}
$$

Given $\zeta \in \mathbb{T}$ define the approach region $\Gamma_{\psi}(\zeta)=\{z \in \mathbb{D}: \psi(|z-\zeta|) \leq$ $1-|z|\}$.

Theorem 2.6. If $\Lambda$ is a sampling set for $\mathcal{N}$ then

$$
\sum_{\lambda \in \Lambda \cap \Gamma_{\psi}(\zeta)} 1-|\lambda|=\infty \quad \text { for all } \zeta \in \mathbb{T} \text { and all } \psi \in \mathcal{F} \text {. }
$$

The results of Section 4.3 show that this is not always sufficient, not even when $\Lambda$ is a sequence. 
We first give a reformulation of Example 6.4 in [5], where we look at when the outside of an approach region $\Gamma_{\psi}(\zeta)$ is, or is not, Hayman-Lyons. All computations should be done in the disc, depending only on what happens in a neighbourhood of a point $\zeta$ on the boundary. Passing to the upper half-plane $U$ with the standard conformal mapping, we may perform the corresponding computations in a disc of fixed radius centered at any point of the real axis.

Lemma 2.7. Let $\psi:(0, \infty) \longrightarrow(0,1]$ be a non-decreasing continuous function with $\psi(0)=0$. The set $\mathcal{D}_{\psi}:=\{x+i y \in \mathbb{C}: 0<y<\psi(|x|)$ or $y \geq 1\}$ is a Hayman-Lyons set if and only if

$$
\int_{0} \frac{\psi(x)}{x^{2}} d x=\infty
$$

Furthermore, if $\mathcal{D}_{\psi}$ is not a Hayman-Lyons set, there exists a harmonic function $h \in \operatorname{Har}_{ \pm}(U)$, non-positive on $\mathcal{D}_{\psi}$, and with $\liminf _{y \rightarrow 0} y h(i y)>0$.

Proof. For any point $\zeta \in \mathbb{R}$ except the origin (but including the point at infinity), $\mathcal{D}_{\psi}$ contains a half-disc centered at $\zeta$, so that the integral $I\left(\mathcal{D}_{\psi}^{\delta}, \zeta, \delta\right) \geq I\left(\mathcal{D}_{\psi}, \zeta, \delta\right)=\infty$. There remains the case $\zeta=0$.

A direct computation shows that

$$
I\left(\mathcal{D}_{\psi}, 0,0\right)=\int_{\mathcal{D}_{\psi}} \frac{d x d y}{x^{2}+y^{2}} \simeq \int_{0}^{1} \int_{0}^{\psi(x)} \frac{1}{x^{2}+y^{2}}=\int_{0}^{1} \frac{\arctan (\psi(x) / x)}{x} d x,
$$

which is finite if and only if $\int_{0} \psi(x) / x^{2} d x<\infty$. Hence $I\left(\mathcal{D}_{\psi}, 0,0\right)=\infty$ when $\int_{0} \psi(x) / x^{2} d x=\infty$.

This same estimate shows that in order to prove that $I\left(\mathcal{D}_{\psi}, 0, \delta\right)<\infty$ when $\int_{0} \psi(x) / x^{2} d x<\infty$ it is enough to see that for any $\delta>0$, there exists a non-decreasing function $\psi_{\delta} \geq 0$ with $\psi_{\delta}(0)=0$ and such that

(i) $\mathcal{D}_{\psi}^{\delta} \subset \mathcal{D}_{\psi_{\delta}}$

(ii) $\int_{0} \psi_{\delta}(x) / x^{2} d x \simeq \int_{0} \psi(x) / x^{2} d x$.

In the construction of $\psi_{\delta}$ only the behavior near zero is relevant, hence we restrict our attention to $x \in[0,1 / 2]$. Recall that the pseudohyperbolic distance between two points $z, \zeta \in U$ is given by $\rho(z, \zeta)=|z-\zeta| /|z-\bar{\zeta}|$.

Let $\eta>0$ (to be chosen later) and consider the function

$$
\psi_{\eta}(x)=\sum_{n=0}^{\infty} \frac{1+\eta}{1-\eta} \psi\left(2^{-n}\right) \chi_{\left[2^{-(n+2)}, 2^{-(n+1)}\right)}(x) .
$$

This corresponds to "raising" the value of $\psi$ at $x=2^{-n}$ by $\eta$ (in the pseudohyperbolic metric) and assigning it to the whole interval $\left[2^{-(n+2)}, 2^{-(n+1)}\right)$ (see Figure 2). 


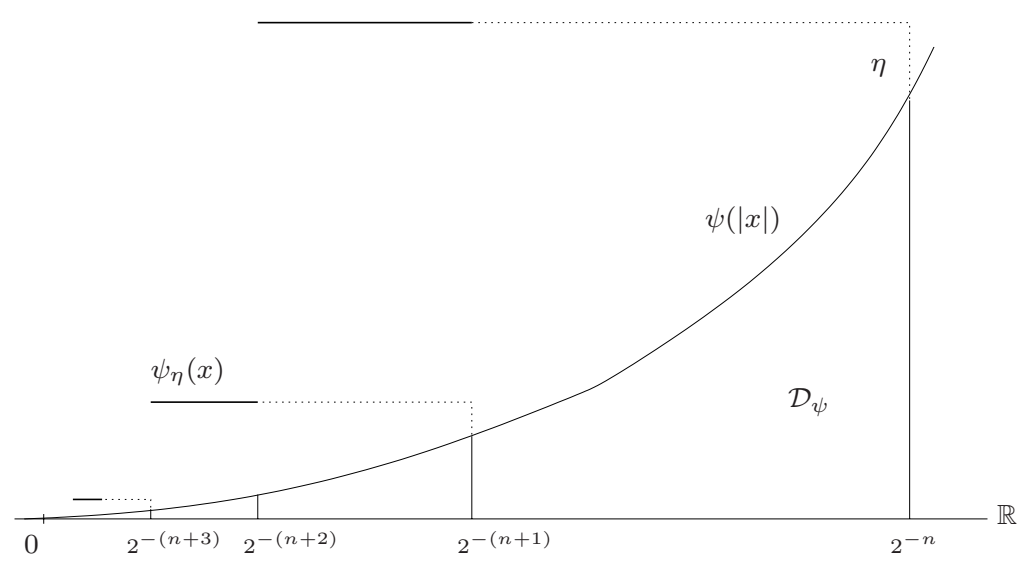

Figure 2 .

It is clear that $\psi_{\eta}$ satisfies (ii) for any $\eta>0$ :

$$
\int_{0} \frac{\psi_{\eta}(x)}{x^{2}} d x=\frac{1+\eta}{1-\eta} \sum_{n=0}^{\infty} \psi\left(2^{-n}\right) \int_{2^{-(n+2)}}^{2^{-(n+1)}} \frac{d x}{x^{2}} \simeq \sum_{n=0}^{\infty} \frac{\psi\left(2^{-n}\right)}{2^{-n}} \simeq \int_{0} \frac{\psi(x)}{x^{2}} d x .
$$

Let us see that the pseudohyperbolic distance between the graph of $\psi_{\eta}$ and the graph of $\psi$ is bigger than $\delta$ if $\eta$ is big enough, and therefore (i) holds as well. In the vertical direction it is clear that we only need to take $\eta \geq \delta$, by construction of $\psi_{\eta}$. For the horizontal direction we have, for any $x \in\left[2^{-(n+1)}, 2^{-n}\right)$,

$$
\begin{aligned}
& \rho\left(\left(2^{-(n+2)}, \psi_{\eta}\left(2^{-(n+2)}\right)\right),(x, \psi(x))\right)= \\
& \quad=\frac{\left|x-2^{-(n+2)}+i\left(\psi(x)-\frac{1+\eta}{1-\eta} \psi\left(2^{-(n+1)}\right)\right)\right|}{\left|x-2^{-(n+2)}+i\left(\psi(x)+\frac{1+\eta}{1-\eta} \psi\left(2^{-(n+1)}\right)\right)\right|} \geq \frac{1}{\left|1+i \frac{1+\eta}{1-\eta} \frac{\psi(x)+\psi\left(2^{-(n+1)}\right)}{x-2^{-(n+2)}}\right|}
\end{aligned}
$$

This is clearly bounded below, since the integrability condition on $\psi$ gives in particular

$$
\lim _{n \rightarrow \infty} \frac{\psi(x)+\psi\left(2^{-(n+1)}\right)}{x-2^{-(n+2)}} \leq \lim _{n \rightarrow \infty} \frac{2 \psi\left(2^{-n}\right)}{2^{-(n+1)}-2^{-(n+2)}}=8 \lim _{n \rightarrow \infty} \frac{\psi\left(2^{-n}\right)}{2^{-n}}=0 .
$$

In the case where the integral is convergent, denoting temporarily $P_{x+i y}(t)$ $:=\frac{1}{\pi} \frac{y}{(x-t)^{2}+y^{2}}$, let

$$
h(x+i y):=P_{x+i y}(0)-C h_{\psi}(x+i y),
$$

where $C>0$ and $h_{\psi}$ is the Poisson integral of the integrable function $\psi(t) / t^{2}$ restricted to the interval $[-1,1]$.

It is well known that the growth of the Poisson integral of an integrable function is such that $\lim _{y \rightarrow 0} y h_{\psi}(x+i y)=0$ (see for instance [11, Coroll. 2]), thus it will be enough to prove that for $C>0$ sufficiently big $h \leq 0$ on $\mathcal{D}_{\psi}$. 
This will be done as soon as we see that $h \leq 0$ for points $z=x_{0}+i y_{0} \in$ $\partial \mathcal{D}_{\psi}$. Since $\lim _{x \rightarrow 0} \psi(x) / x=0$, we have then

$$
P_{z}(0)=\frac{\psi\left(x_{0}\right)}{x_{0}^{2}+\psi^{2}\left(x_{0}\right)} \simeq \frac{\psi\left(x_{0}\right)}{x_{0}^{2}} .
$$

On the other hand

$$
\begin{aligned}
h_{\psi}\left(x_{0}+i y_{0}\right) & \succeq \int_{\left|x-x_{0}\right|<\psi\left(x_{0}\right)} \frac{1}{\psi\left(x_{0}\right)} \frac{\psi(x)}{x^{2}} d x \succeq \frac{1}{\psi\left(x_{0}\right)} \int_{x_{0}}^{x_{0}+\psi\left(x_{0}\right)} \frac{\psi(x)}{x^{2}} d x \\
& \geq\left(\frac{1}{x_{0}}-\frac{1}{x_{0}+\psi\left(x_{0}\right)}\right)=\frac{\psi\left(x_{0}\right)}{x_{0}^{2}} \frac{1}{1+\psi\left(x_{0}\right) / x_{0}} \simeq \frac{\psi\left(x_{0}\right)}{x_{0}^{2}},
\end{aligned}
$$

hence with $C$ big enough we get the desired estimate.

Proof of Theorem 2.6. Assume that there exist $\psi \in \mathcal{F}$ and $\zeta \in \mathbb{T}$ such that $\sum_{\lambda \in \Lambda \cap \Gamma_{\psi}(\zeta)} 1-|\lambda|<\infty$. Similarly to Example 1, consider the function $f \in \mathcal{N}$ such that

$$
\log |f|=\log \left|B_{Z}\right|+h_{\psi},
$$

where $B_{Z}$ is the Blaschke product associated to $Z=\Lambda \cap \Gamma_{\psi}(\zeta)$ and $h_{\psi}$ is the harmonic function obtained by transporting to the disk the function given by the previous lemma.

It is clear then that $f$ is bounded on $\Lambda$. But by the properties of $h_{\psi}$ and by Lemma 2.5 we see that $f$ cannot be bounded in the whole disk. Thus $\Lambda$ is not a determination set for $\mathcal{N}$.

\section{General conditions}

In order to see what extra conditions are required on a Hayman-Lyons set $\Lambda$ to be a determination set for $\mathcal{N}$, assume that $f \in \mathcal{N}$ is such that $\sup _{\Lambda}|f| \leq 1$. According to Remark 2, there exist a Blaschke product $B$ (with zero-sequence $Z$ ) and $F \in \operatorname{Har}_{ \pm}(\mathbb{D})$ such that $\log |f|=\log |B|+F$.

It will enough to see that $F$ is quasi-bounded, that is, that $f$ belongs to the Smirnov class. This is so because the Hayman-Lyons condition implies that $|N T(\Lambda)|=2 \pi$, and we deduce then from Theorem 2.3 that $\sup _{\Lambda}|f|=$ $\sup _{\mathbb{D}}|f|$ (see Remark 1$)$.

The hypothesis is, in these terms,

$$
F(\lambda) \leq \log \frac{1}{|B(\lambda)|} \quad \lambda \in \Lambda
$$

and we would like to impose certain conditions on $\Lambda$ so that this estimate implies that $F$ has a quasi-bounded harmonic majorant. 
A first observation is that the zeros of $B$ far from a given $\lambda$ are no obstruction to such a majorization. The following is a restatement of $[6$, Proposition 4.1, pp. 13-14], and of part of its proof.

Lemma 3.1. Let $B$ be the Blaschke product associated to a Blaschke sequence $Z$. For any $\delta \in(0,1)$, there exists a positive quasi-bounded harmonic function $H_{B}=P[w], w \in L^{1}(\mathbb{T})$, such that $-\log |B(z)| \leq H_{B}(z)-$ $\sum_{a \in Z \cap D(z, \delta)} \log \rho(z, a)$, for any $z \in \mathbb{D}$. Furthermore

$$
w(\zeta)=c_{0} \sum_{a \in Z} \chi_{I_{a}}(\zeta)
$$

where $c_{0}$ is an appropriate positive constant, and $I_{a}=\left\{\zeta \in \mathbb{T}:\left|\zeta-\frac{a}{|a|}\right| \leq\right.$ $1-|a|\}$ is the "Privalov shadow" of a on $\mathbb{T}$.

Given $\delta \in(0,1)$, there exists thus $H_{B}$ harmonic, quasi-bounded and positive such that,

$$
F(\lambda) \leq H_{B}(\lambda)+\sum_{a \in Z \cap D(\lambda, \delta)} \log \frac{1}{\rho(\lambda, a)} \quad \lambda \in \Lambda .
$$

It is clear that we cannot expect to bound the local sum in the right hand side of this inequality by a quasi-bounded harmonic function on all $\lambda \in \Lambda$ (it could happen, for example, that $Z \cap \Lambda \neq \emptyset$ ). Rather, we would like to find conditions on $\Lambda$ that ensure such a bound for a subset $\Lambda^{\prime} \subset \Lambda$ big enough so that the estimate of $F$ by a quasi-bounded harmonic function on $\Lambda^{\prime}$ implies the same estimate everywhere (in the spirit of the Hayman-Lyons condition for functions in $\left.\operatorname{Har}_{ \pm}(\mathbb{D})\right)$.

For that purpose we need a measure of the "vulnerability" of $\Lambda$ to the presence of zeros of a Blaschke product.

Consider the dyadic squares $\left\{Q_{n, k}\right\}_{(n, k) \in \mathcal{I}}$ defined in (2.4) and denote by $\tilde{Q}_{n, k}$ the union of all $Q_{m, j}$ such that $\overline{Q_{n, k}} \cap \overline{Q_{m, j}} \neq \emptyset$. There exists $\delta_{0}>0$ such that the dilation ${\overline{Q_{n, k}}}_{0}$ is contained in $\tilde{Q}_{n, k}$ for all $(n, k) \in \mathcal{I}$.

To measure the vulnerability of $\Lambda$ at each $Q_{n, k}$, for $N \in \mathbb{N}$ and $\delta \in(0,1)$ consider

$$
w_{n, k}(\Lambda, N)=\sup \left\{\left(\inf _{\lambda \in \Lambda \cap \overline{Q_{n, k}}} \sum_{j=1}^{N} \log \frac{1}{\rho\left(\lambda, a_{j}\right)}\right), a_{1}, \ldots, a_{N} \in \overline{Q_{n, k}} \delta\right\} .
$$

We take an empty sum to be 0 , so that $w_{n, k}(\Lambda, 0)=0$ for any set $\Lambda$. Clearly, $w_{n, k}(\Lambda, N)$ is an increasing function of $N$, and there exists $C(\delta)>0$ such that $w_{n, k}(\Lambda, N) \geq N C(\delta)$. 
Given a Blaschke sequence $Z$ let $N_{n, k}=\#\left(Z \cap \tilde{Q}_{n, k}\right)$. The Blaschke condition is thus equivalent to $\sum_{n} 2^{-n} \sum_{k=0}^{2^{n}-1} N_{n, k}<\infty$. Any sequence of integers $\left\{N_{n, k}\right\}_{(n, k) \in \mathcal{I}}$ satisfying this condition will be called a Blaschke distribution.

Theorem 3.2. Let $\Lambda \subset \mathbb{D}$. Each of the following properties implies the next one.

(a) For any Blaschke distribution $\left\{N_{n, k}\right\}_{(n, k) \in \mathcal{I}}$, there exists $\mathcal{Q} \subset \mathcal{I}$ such that

$$
\begin{aligned}
\Lambda \cap Q_{n, k} \neq \emptyset \quad \text { for any }(n, k) & \in \mathcal{Q}, \\
\sum_{(n, k) \in \mathcal{Q}} 2^{-n} P_{c_{n, k}}(\zeta) & =\infty
\end{aligned}
$$

for all $\zeta \in \mathbb{T}$, and

$$
\sum_{(n, k) \in \mathcal{Q}} 2^{-n} w_{n, k}\left(\Lambda, N_{n, k}\right)<\infty
$$

(b) For any Blaschke distribution $\left\{N_{n, k}\right\}_{(n, k) \in \mathcal{I}}$ and any positive finite measure $\nu$ on $\mathbb{T}$, singular with respect to the Lebesgue measure, there exists $\mathcal{Q} \subset \mathcal{I}$ satisfying (3.2), (3.4) and condition (3.3) almost everywhere with respect to $\nu$.

(c) $\Lambda$ is a determination set for the Nevanlinna class.

(d) For any Blaschke distribution $\left\{N_{n, k}\right\}_{(n, k) \in \mathcal{I}}$ and any positive finite measure $\nu$ on $\mathbb{T}$, singular with respect to the Lebesgue measure, there exists $\mathcal{Q} \subset \mathcal{I}$ satisfying (3.2), (3.4) and

$$
\int_{\mathbb{T}} \sum_{(n, k) \in \mathcal{Q}} 2^{-n} P_{c_{n, k}}(\zeta) d \nu(\zeta)=\infty
$$

(e) For any Blaschke distribution $\left\{N_{n, k}\right\}_{(n, k) \in \mathcal{I}}$ and any $\zeta \in \mathbb{T}$, there exists $\mathcal{Q} \subset \mathcal{I}$ satisfying (3.2), (3.3) and (3.4).

As pointed out before the statement of Theorem A, condition (3.3) is precisely the Hayman-Lyons condition for the set $\Lambda \cap\left\{Q_{n, k}\right\}_{(n, k) \in \mathcal{Q}}$.

We will see in the next Section how these somewhat cumbersome conditions can be used to provide precise geometric conditions, at least when then set $\Lambda$ has some regularity.

Open question: are there examples of sets $\Lambda$ to show that the first and last of those properties are actually different? 
Proof. (a) $\Rightarrow$ (b). Obvious.

(b) $\Rightarrow$ (c). Start, as in the general scheme, with $f \in \mathcal{N}$ such that $\sup _{\Lambda}|f| \leq 1$ and consider the decomposition $\log |f|=\log |B|+F$. By the Riesz-Smirnov factorization (2.1), the function $F$ can be written as

$$
F=h_{1}-h_{2}+H_{1}-H_{2},
$$

where $h_{i}, H_{i} \in \operatorname{Har}_{+}(\mathbb{D}), H_{i}$ are quasi-bounded and $h_{i}=P\left[\nu_{i}\right]$, with $\nu_{i}$ positive finite measure in $\mathbb{T}$, singular with respect to the Lebesgue measure.

In order to see that $f \in \mathcal{N}^{+}$it will be enough to prove that $h_{1}$ has a quasi-bounded majorant. To do that we use (b) with the singular measure $\nu_{1}$ and the Blaschke distribution determined by $B$. Let $\mathcal{Q} \subset \mathcal{I}$ be the set of indices for which (b) holds.

Let $g \in \mathcal{N}$ be such that $\log |g|=\log |f|-H_{1}=\log |B|+h_{1}-h_{2}-H_{2}$ (explicitly $g=f e^{-\left(H_{1}+i \tilde{H}_{1}\right)}$, where $\tilde{H}_{1}$ denotes the harmonic conjugate of $\left.H_{1}\right)$. Then, the corresponding estimate (3.1) for $g$ becomes

$$
h_{1}(\lambda)-h_{2}(\lambda)-H_{2}(\lambda) \leq H_{B}(\lambda)+\sum_{a \in Z \cap D(\lambda, \delta)} \log \frac{1}{\rho(\lambda, a)} \quad \lambda \in \Lambda,
$$

for some positive quasi-bounded harmonic function $H_{B}$.

For each $(n, k) \in \mathcal{Q}$ there is a particular $j=j(n, k)$ such that $\lambda_{j(n, k)} \in$ $\Lambda \cap \overline{Q_{n, k}}$ and

$$
\sum_{a \in Z \cap Q_{n, k}^{\delta}} \log \frac{1}{\rho\left(\lambda_{j(n, k)}, a\right)}=\inf _{\lambda_{j} \in \Lambda \cap \overline{Q_{n, k}}} \sum_{a \in Z \cap Q_{n, k}^{\delta}} \log \frac{1}{\rho\left(\lambda_{j}, a\right)} \leq w_{n, k}\left(\Lambda, N_{n, k}\right) .
$$

Let $I_{n, k}$ be the dyadic arcs defined in (2.3) and let $C>0$. According to (3.4) the function $H_{L}=P[W]$, with

$$
W:=C \sum_{(n, k) \in \mathcal{Q}} w_{n, k}\left(\Lambda, N_{n, k}\right) \chi_{I_{n, k}},
$$

is a positive quasi-bounded harmonic function. The usual estimate of the Poisson kernel by a "square" kernel (or a direct computation) shows that $P\left[\chi_{I_{z}}\right](z) \geq c>0$, with $c$ independent of $z$. Hence for $C$ well chosen,

$$
w_{n, k}\left(\Lambda, N_{n, k}\right) \leq H_{L}\left(\lambda_{j(n, k)}\right), \quad(n, k) \in \mathcal{Q} .
$$

Then, defining $\Lambda^{\prime}=\left\{\lambda_{j(n, k)}\right\}_{(n, k) \in \mathcal{Q}}$ we have $h_{1}-h_{2}-H_{2} \leq H_{B}+H_{L}$ on $\Lambda^{\prime}$. Condition (3.3) being satisfied $\nu_{1}$-a.e. and [3, Theorem 2] show then that

$$
1 \leq \inf _{\Lambda^{\prime}} \frac{h_{2}+H_{2}+H_{B}+H_{L}}{h_{1}}=\inf _{\mathbb{D}} \frac{h_{2}+H_{2}+H_{B}+H_{L}}{h_{1}} .
$$

Thus $h_{1}-h_{2}-H_{2} \leq H_{B}+H_{L}$ everywhere, and $\log |f| \leq \log |B|+H_{1}+$ $H_{B}+H_{L}$, as desired. 
$(c) \Rightarrow(d)$. Following the original ideas of Beurling [1], and similarly to the proof of Lemma 1 in [5], for a given set of indices $\mathcal{Q} \subset \mathcal{I}$ satisfying (3.2) and for $(n, k) \in \mathcal{Q}$, let $\lambda_{n, k} \in \Lambda \cap Q_{n, k}$, and define the (possibly divergent) series

$$
H_{\nu}(z):=\sum_{(n, k) \in \mathcal{Q}} 2^{-n} P[\nu]\left(\lambda_{n, k}\right) P_{z}\left(\lambda_{n, k}^{*}\right),
$$

where $\lambda_{n, k}^{*}=\lambda_{n, k} /\left|\lambda_{n, k}\right|$.

The terms of this series are positive harmonic functions so by Harnack's theorem $H_{\nu}$ is either identically $+\infty$ or it defines a positive harmonic function.

Suppose that $\mathcal{Q}$ is a set for which (3.5) fails. Then the series defining $H_{\nu}(0)$ converges, and therefore $H_{\nu}$ is a positive harmonic function. Notice also that $\lim _{|z| \rightarrow 1}(1-|z|) H_{\nu}(z)=0$, since each term of the sum has this property, and we can apply dominated convergence.

By retaining only the $(n, k)$ term of the sum, we see that

$$
H_{\nu}\left(\lambda_{n, k}\right) \succeq 2^{-n} P[\nu]\left(\lambda_{n, k}\right) \frac{1}{1-\left|\lambda_{n, k}\right|} \succeq P[\nu]\left(\lambda_{n, k}\right) .
$$

Thus, using Harnack's inequality and choosing an appropriate constant $C_{0}>0$, we obtain a function

$$
h_{\nu}:=P[\nu]-C_{0} H_{\nu}
$$

which is non-positive on $\bigcup_{(n, k) \in \mathcal{Q}} Q_{n, k}$, and tends to infinity as $z$ tends radially to the boundary a.e. with respect to $\nu$.

Now suppose that (d) doesn't hold. This means that we are given a Blaschke distribution $\left\{N_{n, k}\right\}_{(n, k) \in \mathcal{I}}$ and a singular measure $\nu$ such that for any $\mathcal{Q}$ such that (3.4) holds, then (3.5) fails.

Claim. There exist a constant $\gamma>0$ and a subset $\Lambda_{0} \subset \Lambda$ such that

(i) $\sum_{(n, k):\left(\Lambda \backslash \Lambda_{0}\right) \cap Q_{n, k} \neq \emptyset} 2^{-n} P[\nu]\left(\lambda_{n, k}\right) \simeq \sum_{(n, k):\left(\Lambda \backslash \Lambda_{0}\right) \cap Q_{n, k} \neq \emptyset} 2^{-n} \int_{\mathbb{T}} P_{c_{n, k}}(\zeta) d \nu(\zeta)<\infty$,

(ii) $\gamma P[\nu]\left(\lambda_{n, k}\right) \leq w_{n, k}\left(\Lambda, N_{n, k}\right)$ for any $(n, k)$ with $\Lambda_{0} \cap Q_{n, k} \neq \emptyset$.

To see this, define

$$
\mathcal{L}_{j}:=\left\{(n, k) \in \mathcal{I}: \Lambda \cap Q_{n, k} \neq \emptyset \text { and } w_{n, k}\left(\Lambda, N_{n, k}\right) \leq 2^{-j} P[\nu]\left(\lambda_{n, k}\right)\right\} .
$$

If there exists some $j_{0}$ such that

$$
\sum_{(n, k) \in \mathcal{L}_{j_{0}}} 2^{-n} P[\nu]\left(\lambda_{n, k}\right)<\infty
$$

then define $\Lambda \backslash \Lambda_{0}:=\Lambda \cap \bigcup_{(n, k) \in \mathcal{L}_{j_{0}}} Q_{n, k}$, and we have the result with $\gamma=2^{-j_{0}}$. 
Otherwise, set $j_{1}=1$ and define recursively $j_{m+1}>j_{m}$ and subsets $\mathcal{A}_{m} \subset \mathcal{L}_{j_{m}}$ such that

- $\mathcal{A}_{m+1} \cap \mathcal{A}_{l}=\emptyset, 1 \leq l \leq m$, and

- $1 \leq \sum_{(n, k) \in \mathcal{A}_{m}} 2^{-n} P[\nu]\left(\lambda_{n, k}\right) \leq M$.

This is possible because the terms to be summed belong to a divergent series, and are all bounded by a constant. Now, taking $\mathcal{Q}:=\cup \mathcal{A}_{m}$, we have

$$
\begin{aligned}
\sum_{(n, k) \in \mathcal{Q}} 2^{-n} w_{n, k}\left(\Lambda, N_{n, k}\right) & \leq \sum_{m} 2^{-j_{m}}\left(\sum_{(n, k) \in \mathcal{A}_{m}} 2^{-n} P[\nu]\left(\lambda_{n, k}\right)\right) \\
& \leq M \sum_{m} 2^{-j_{m}}<\infty
\end{aligned}
$$

while

$$
\sum_{(n, k) \in \mathcal{Q}} 2^{-n} P[\nu]\left(\lambda_{n, k}\right)=\infty
$$

which contradicts the hypothesis. The claim is proved.

We now proceed to prove that the set $\Lambda$ is not of determination for the Nevalinna class. Let $h_{\nu}$ be the function constructed above, using the set $\Lambda \backslash \Lambda_{0}$ as the set which doesn't satisfy (3.5). Let $B$ be a Blaschke product with $N_{n, k}$ zeros $b_{1}, \ldots, b_{N_{n, k}}$ located in $Q_{n, k}^{\delta}$ chosen as the solution to the extremal problem in the definition of $w_{n, k}$ :

$$
\begin{aligned}
\inf _{\lambda \in \Lambda \cap \bar{Q}_{n, k}} \sum_{j=1}^{N_{n, k}} \log \frac{1}{\rho\left(\lambda, b_{j}\right)} & =\sup _{a_{1, \ldots, a_{N, k}} \in Q_{n, k}^{\delta}} \inf _{\lambda \in \Lambda \cap \bar{Q}_{n, k}} \sum_{j=1}^{N_{n, k}} \log \frac{1}{\rho\left(\lambda, a_{j}\right)} \\
& =w_{n, k}\left(\Lambda, N_{n, k}\right) .
\end{aligned}
$$

Choose an integer $m$ such that $m \gamma \geq 1$ and pick a function $f \in \mathcal{N}$ with

$$
\log |f|=m \log |B|+h_{\nu} .
$$

By construction $h_{\nu} \leq 0$ on $\Lambda \backslash \Lambda_{0}$ and $h_{\nu} \leq \mathcal{P}[\nu]$ on $\Lambda$. Also, (ii) implies that $\log |f| \leq 0$ on $\Lambda_{0}$, so altogether $\log |f| \leq 0$ on the whole of $\Lambda$. On the other hand the fact that

$$
\limsup _{|z| \rightarrow 1}(1-|z|)\left[\log |B(z)|+H_{\nu}(z)\right]=0
$$

shows that $f$ cannot be bounded on the disk.

$(\mathrm{d}) \Rightarrow(\mathrm{e})$. Condition (e) is the special case of $(\mathrm{d})$ where $\nu$ is a point mass. 


\section{Regular sampling sequences}

In this section we give precise conditions for three types of regular sets to be sampling for $\mathcal{N}$.

\subsection{Fine nets}

Let $g:(0,1] \longrightarrow(0,1]$ be a non-decreasing continuous function with $g(0)=0$. A sequence $\Lambda$ is called a $g$-net if and only if

(i) The disks $D(\lambda, g(1-|\lambda|)), \lambda \in \Lambda$, are mutually disjoint,

(ii) There exists $C>0$ such that $\bigcup_{\lambda \in \Lambda} D(\lambda, C g(1-|\lambda|))=\mathbb{D}$.

We characterize sampling $g$-nets in terms of the growth of $g$.

Theorem 4.1. Let $\Lambda$ be a g-net. The following properties are equivalent:

(a) $\Lambda$ is a sampling sequence for $\mathcal{N}$.

(b) Condition (2.5) holds.

(c) $\int_{0} \frac{d t}{t^{1 / 2} g(t)}=\infty$.

Remark 3. The conditions above can be formulated also in terms of the number $M_{n, k}$ of points of $\Lambda$ in a dyadic square $Q_{n, k}$. In this case $M_{n, k}$ is essentially independent of $k$, in the sense that there exist $M_{n}$ and a constant $C>0$ such that $C^{-1} M_{n} \leq M_{n, k} \leq C M_{n}$ for all $0 \leq k<2^{n}$. Then the conditions in the theorem above are equivalent to $\sum_{n}\left(M_{n} 2^{-n}\right)^{1 / 2}=\infty$ (see Lemma 4.2).

Proof of Theorem 4.1. (a) $\Rightarrow$ (b) is a particular case of Theorem 2.6.

$(\mathrm{b}) \Rightarrow(\mathrm{c})$. It will be enough to prove the following lemma.

Lemma 4.2. Assume that $\Lambda$ is a g-net satisfying condition (2.5). Let $M_{n, k}=\# \Lambda \cap Q_{n, k}$ and assume that there exist $C>0$ and positive integers $M_{n}$ such that $C^{-1} M_{n} \leq M_{n, k} \leq C M_{n}$. Then

$$
\sum_{n}\left(M_{n} 2^{-n}\right)^{1 / 2}=\infty
$$

A volume estimate shows that $M_{n} \simeq\left(g\left(2^{-n}\right)\right)^{-2}$, and therefore

$$
\int_{0} \frac{d t}{t^{1 / 2} g(t)} \simeq \sum_{n} 2^{-n / 2} \frac{1}{g\left(2^{-n}\right)} \simeq \sum_{n}\left(M_{n} 2^{-n}\right)^{1 / 2}=\infty
$$


Proof. We want to prove that if $\sum_{n}\left(M_{n} 2^{-n}\right)^{1 / 2}<\infty$ there exists $\psi \in \mathcal{F}$ such that $\sum_{\lambda \in \Lambda \cap \Gamma_{\psi}(\zeta)} 1-|\lambda|<\infty$, thus contradicting (2.5).

It will be expedient to write the computation in the upper half-plane $U$ after a conformal mapping. Calling again the resulting sets $\Lambda$ and $\Gamma_{\psi}(\zeta)$, we need to prove that there exists $\psi \in \mathcal{F}$ such that

$$
\sum_{\lambda \in \Lambda \cap \Gamma_{\psi}(\zeta)} 1-|\lambda| \simeq \sum_{n \geq 0} 2^{-n} \#\left(\Lambda \cap \Gamma_{\psi}(\zeta) \cap\left\{2^{-n-1}<y \leq 2^{-n}\right\}\right)<\infty
$$

Since $\psi$ is an increasing function, the set $\Gamma_{\psi}(\zeta) \cap\left\{2^{-n-1}<y \leq 2^{-n}\right\}$ is contained in the rectangle $\left\{|x| \leq \psi^{-1}\left(2^{-n}\right), 2^{-n-1}<y \leq 2^{-n}\right\}$. Therefore, splitting the sum for the different $Q_{n, k}$ and using that $M_{n} \simeq g^{-2}\left(2^{-n}\right)$ we have

$$
\begin{aligned}
\sum_{\lambda \in \Lambda \cap \Gamma_{\psi}(\zeta)} 1-|\lambda| & \preceq \sum_{n \geq 0} 2^{-n} \sum_{k: k 2^{-n} \leq \psi^{-1}\left(2^{-n}\right)} M_{n, k} \preceq \sum_{n \geq 0} M_{n} \psi^{-1}\left(2^{-n}\right) \\
& \simeq \sum_{n \geq 0} \frac{\psi^{-1}\left(2^{-n}\right)}{g^{2}\left(2^{-n}\right)} .
\end{aligned}
$$

It will be enough to see that $\psi$ defined by $\psi^{-1}(t)=\sqrt{t} g(t)$ is in $\mathcal{F}$, since then

$$
\sum_{\lambda \in \Lambda \cap \Gamma_{\psi}(\zeta)} 1-|\lambda| \preceq \sum_{n \geq 0} \frac{2^{-n / 2}}{g\left(2^{-n}\right)} \simeq \int_{0} \frac{d t}{\sqrt{t} g(t)}<\infty .
$$

By definition $\psi$ is non-decreasing, continuous and $\psi(0)=0$. Also

$$
\begin{aligned}
\int_{0}^{1} \frac{d t}{\sqrt{t} g(t)} & =\int_{0}^{1} \frac{d t}{\psi^{-1}(t)}=\int_{1}^{\infty}\left|\left\{t: 1 / \psi^{-1}(t) \geq \alpha\right\}\right| d \alpha \\
& =\int_{0}^{1}\left|\left\{t: \psi^{-1}(t) \leq s\right\}\right| \frac{d s}{s^{2}}=\int_{0}^{1}|\{t: t \leq \psi(s)\}| \frac{d s}{s^{2}} \\
& =\int_{0}^{1} \frac{\psi(s)}{s^{2}} d s<\infty,
\end{aligned}
$$

as desired.

$(\mathrm{c}) \Rightarrow(\mathrm{a})$. Given a Blaschke sequence $Z$ we want to choose a family of indices $\mathcal{Q} \subset \mathcal{I}$ satisfying the conditions of Theorem 3.2(a). First we need a control of the vulnerability on the squares where $N_{n, k}=\#\left(Z \cap \tilde{Q}_{n, k}\right)$ is small.

Lemma 4.3. If $\Lambda$ is a g-net, there exist $\varepsilon>0, n_{0} \in \mathbb{N}$ and $C>0$ such that whenever $n \geq n_{0}$ and $N \leq \varepsilon \#\left(\Lambda \cap Q_{n, k}\right)$, then $w_{n, k}(\Lambda, N) \leq C N$. 
Proof. Denote $Q=Q_{n, k}, \tilde{Q}=\tilde{Q}_{n, k}$ and $M=\#(\Lambda \cap Q)$. Then $M \simeq$ $g\left(2^{-n}\right)^{-2}$ and there exist constants $c<C$ such that the disks $D\left(\lambda, c g\left(2^{-n}\right)\right)$, $\lambda \in Q$, are mutually disjoint and the disks $D\left(\lambda, C g\left(2^{-n}\right)\right)$ cover the whole set $Q$. Let $Z=\left\{a_{1}, \ldots, a_{N}\right\} \subset \bar{Q}^{\delta}$ and consider

$$
\Lambda_{Q}^{\prime}:=\left\{\lambda \in \Lambda \cap Q: \rho(\lambda, a) \geq C g\left(2^{-n}\right) \text {, for all } a \in Z \cap \tilde{Q}\right\} .
$$

For $\varepsilon$ small enough, and $n$ (and therefore $M$ ) large enough, $\# \Lambda_{Q}^{\prime} \geq M / 2$.

For $\lambda^{\prime} \in \Lambda_{Q}^{\prime}$ and $z \in D\left(\lambda^{\prime}, c g\left(2^{-n}\right)\right)$ we have $\rho\left(z, a_{j}\right) \preceq \rho\left(\lambda^{\prime}, a_{j}\right)$ for all $a_{j} \in \tilde{Q}$, and therefore

$$
\log \frac{1}{\rho\left(\lambda^{\prime}, a_{j}\right)} \preceq \frac{1}{m\left(D\left(\lambda^{\prime}, c g\left(2^{-n}\right)\right)\right.} \int_{D\left(\lambda^{\prime}, c g\left(2^{-n}\right)\right)} \log \frac{1}{\rho\left(z, a_{j}\right)} d m(z) .
$$

Choose $r \in(0,1)$ large enough so that $\bar{Q} \subset D(a, r)$ for any $a \in \tilde{Q}$. Then, for any $a_{1}, \ldots, a_{N} \in \tilde{Q}$,

$$
\begin{aligned}
\sum_{\lambda^{\prime} \in \Lambda_{Q}^{\prime}} \sum_{j=1}^{N} \log \frac{1}{\rho\left(\lambda^{\prime}, a_{j}\right)} & =\sum_{j=1}^{N} \sum_{\lambda^{\prime} \in \Lambda_{Q}^{\prime}} \log \frac{1}{\rho\left(\lambda^{\prime}, a_{j}\right)} \\
& \preceq \sum_{j=1}^{N} \frac{1}{\left(g\left(2^{-n}\right) 2^{-n}\right)^{2}} \int_{D\left(a_{j}, r\right)} \log \frac{1}{\rho\left(z, a_{j}\right)} d m(z),
\end{aligned}
$$

Since $1-\left|a_{j}\right| \simeq 2^{-n}$, applying an automorphism of the disk sending $a_{j}$ to the origin, we see that

$$
\frac{1}{\left(2^{-n}\right)^{2}} \int_{D\left(a_{j}, r\right)} \log \frac{1}{\rho\left(z, a_{j}\right)} d m(z) \preceq \int_{D(0, r)} \log \frac{1}{|z|} d m(z) \leq C .
$$

Finally,

$$
\sum_{\lambda^{\prime} \in \Lambda_{Q}^{\prime}}\left(\sum_{j=1}^{N} \log \frac{1}{\rho\left(\lambda^{\prime}, a_{j}\right)}\right) \preceq \frac{1}{g^{2}\left(2^{-n}\right)} N \simeq M N,
$$

and since $\# \Lambda_{Q}^{\prime} \geq M / 2$, the average value of the summands in the first sum is bounded by a constant multiple of $N$.

For $\varepsilon \in(0,1)$ small enough, define

$$
\mathcal{Q}=\left\{(n, k): N_{n, k} \leq \varepsilon \#\left(\Lambda \cap Q_{n, k}\right)\right\} .
$$

Lemma 4.3 together with the Blaschke condition give (3.4) in Theorem 3.2.

In order to prove (3.3) in Theorem 3.2, for each $(n, k)$ pick $c_{n, k} \in Q_{n, k}$, for instance its center. By rotation invariance of the properties of being a 
$g$-net or a Blaschke sequence we can assume that $\zeta=1$, so it will be enough to see that

$$
\sum_{(n, k) \in \mathcal{Q}}\left(\frac{1-\left|c_{n, k}\right|^{2}}{\left|1-c_{n, k}\right|}\right)^{2}=\infty
$$

Let

$$
L_{n}:=\#\{k:(n, k) \notin \mathcal{Q}\} .
$$

Observe that $\frac{1-|c|^{2}}{|1-c|}$ is bigger when the argument of $c$ in $(-\pi, \pi]$ is closer to 0 . So we must have, for any fixed $n$,

$$
\sum_{k:(n, k) \in \mathcal{Q}}\left(\frac{1-\left|c_{n, k}\right|^{2}}{\left|1-c_{n, k}\right|}\right)^{2} \succeq \sum_{k \geq L_{n}}\left(\frac{2^{-n}}{k 2^{-n}}\right)^{2} \simeq \frac{1}{L_{n}} .
$$

On the other hand

$$
\sum_{k:(n, k) \notin \mathcal{Q}} N_{n, k} \geq \varepsilon \sum_{k:(n, k) \notin \mathcal{Q}} \#\left(\Lambda \cap Q_{n, k}\right) \geq \varepsilon C \frac{L_{n}}{\left(g\left(2^{-n}\right)\right)^{2}},
$$

and the Blaschke condition implies

$$
\sum_{n} \frac{2^{-n} L_{n}}{\left(g\left(2^{-n}\right)\right)^{2}}<\infty
$$

If (4.4) doesn't hold we have $\sum_{n} 1 / L_{n}<\infty$, and

$$
\begin{aligned}
\int_{0} \frac{d t}{t^{1 / 2} g(t)} & \simeq \sum_{n \geq 0} \frac{2^{-n / 2}}{g\left(2^{-n}\right)}=\sum_{n \geq 0} \frac{1}{L_{n}^{1 / 2}} \frac{2^{-n / 2} L_{n}^{1 / 2}}{g\left(2^{-n}\right)} \\
& \leq\left(\sum_{n \geq 0} \frac{1}{L_{n}}\right)^{1 / 2}\left(\sum_{n \geq 0} \frac{2^{-n} L_{n}}{\left(g\left(2^{-n}\right)\right)^{2}}\right)^{1 / 2}<\infty
\end{aligned}
$$

which contradicts the hypothesis.

\subsection{Discretized rings}

Let $r_{m} \in(0,1)$ be an increasing sequence of radii with $\lim _{m} r_{m}=1$ and $\sup _{m} \frac{1-r_{m+1}}{1-r_{m}}<1$. Let $\epsilon_{m}$ be a decreasing sequence of hyperbolic distances such that $\lim _{m} \epsilon_{m}=0$. The discretized rings associated to $\left\{r_{m}\right\}_{m}$ and $\left\{\epsilon_{m}\right\}_{m}$ is the sequence $\Lambda=\left\{\lambda_{m, j}\right\}_{m, j}$, where

$$
\lambda_{m, j}=r_{m} \exp \left(j \frac{2 \pi i}{\left(1-r_{m}\right) \epsilon_{m}}\right) \quad m \in \mathbb{N}, \quad 0 \leq j<\left[\frac{1}{\left(1-r_{m}\right) \epsilon_{m}}\right] .
$$


Theorem 4.4. Let $\Lambda$ be discretized rings associated to $\left\{r_{m}\right\}_{m}$ and $\left\{\epsilon_{m}\right\}_{m}$. The following properties are equivalent:

(a) $\Lambda$ is a sampling sequence for $\mathcal{N}$.

(b) Condition (2.5) holds.

(c) $\sum_{m=0}^{\infty}\left(\frac{1-r_{m}}{\epsilon_{m}}\right)^{1 / 2}=\infty$.

The proof follows the same scheme as the proof of Theorem 4.1.

Proof. $(\mathrm{a}) \Rightarrow(\mathrm{b})$ is a particular case of Theorem 2.6.

(b) $\Rightarrow(c)$. Assume that

$$
\sum_{m}\left(\frac{1-r_{m}}{\epsilon_{m}}\right)^{1 / 2}<\infty .
$$

Consider the sequence $\eta_{m}=\left[\left(1-r_{m}\right) \epsilon_{m}\right]^{1 / 2}$ decreasing to zero. Let $\psi$ be a continuous, non-decreasing function such that for all $m \in \mathbb{N}, \psi\left(\eta_{m}\right)=1-r_{m}$ and

$$
\int_{\eta_{m+1}}^{\eta_{m}} \frac{\psi(x)}{x^{2}} d x \leq 2 \int_{\eta_{m+1}}^{\eta_{m}} \frac{1-r_{m+1}}{x^{2}} d x
$$

It is clear that $\psi \in \mathcal{F}$ :

$$
\begin{aligned}
\int_{0} \frac{\psi(x)}{x^{2}} d x & \preceq \sum_{m \geq 1} \int_{\eta_{m+1}}^{\eta_{m}} \frac{1-r_{m+1}}{x^{2}} d x \simeq \sum_{m \geq 1}\left(1-r_{m+1}\right)\left(\frac{1}{\eta_{m+1}}-\frac{1}{\eta_{m}}\right) \\
& \leq \sum_{m \geq 1} \frac{1-r_{m+1}}{\eta_{m+1}}=\sum_{m \geq 1}\left(\frac{1-r_{m+1}}{\epsilon_{m+1}}\right)^{1 / 2}<\infty
\end{aligned}
$$

On the other hand, a length estimate shows that the number of $j$ such that $\lambda_{m, j} \in \Gamma_{\psi}(\zeta)$ is approximately $\frac{\psi^{-1}\left(1-r_{m}\right)}{\left(1-r_{m}\right) \epsilon_{m}}$. Since $\psi^{-1}\left(1-r_{m}\right)=\eta_{m}$ we have

$$
\begin{aligned}
\sum_{\lambda \in \Lambda \cap \Gamma_{\psi}(\zeta)} 1-|\lambda| & \simeq \sum_{m \geq 0}\left(1-r_{m}\right) \frac{\psi^{-1}\left(1-r_{m}\right)}{\left(1-r_{m}\right) \epsilon_{m}}=\sum_{m \geq 0} \frac{\eta_{m}}{\epsilon_{m}} \\
& =\sum_{m \geq 0}\left(\frac{1-r_{m}}{\epsilon_{m}}\right)^{1 / 2}<\infty
\end{aligned}
$$

which contradicts (b).

$(\mathrm{c}) \Rightarrow(\mathrm{a})$. Given a Blaschke sequence $Z$, we want to choose a family of indices $\mathcal{Q}$ satisfying the conditions of Theorem $3.2(\mathrm{a})$. 
We begin by showing that Lemma 4.3 still holds for discretized rings.

Proof of Lemma 4.3 for discretized rings. Let $Q=Q_{n, k}, \tilde{Q}=\tilde{Q}_{n, k}$ and $M=\#(\Lambda \cap Q)$.

Since $\sup _{n} \frac{1-r_{n+1}}{1-r_{n}}<1$, there is at most a finite number of $r_{m}$ with $2^{-(n+1)}<1-r_{m} \leq 2^{-n}$. There is no loss of generality in assuming that there is just one such $r_{m}$, and therefore $M \simeq 1 / \epsilon_{m}$. In particular, there exist $c<C$ such that $D\left(\lambda, c \epsilon_{m}\right), \lambda \in Q$, are mutually disjoint and $D\left(\lambda, C \epsilon_{m}\right)$ cover the segment $\left\{z:|z|=r_{m}\right\} \cap Q$.

Given $Z=\left\{a_{1}, \ldots, a_{N}\right\} \subset \bar{Q}^{\delta}$ consider now

$$
\Lambda_{Q}^{\prime}:=\left\{\lambda \in \Lambda \cap Q: \rho(\lambda, a) \geq C \epsilon_{m} \text {, for all } a \in Z \cap \tilde{Q}\right\} .
$$

Again, for suitable $C, m$ and $r \in(0,1)$, we have $\# \Lambda_{Q}^{\prime} \geq M / 2$ and $\bar{Q} \subset$ $D(a, r)$ for any $a \in \tilde{Q}$. We proceed as before, but replacing the area averages in (4.2) by the line averages. For $\lambda^{\prime} \in \Lambda_{Q}^{\prime}$,

$$
\begin{aligned}
\log \frac{1}{\rho\left(\lambda^{\prime}, a_{j}\right)} & \preceq \frac{1}{\left|J\left(\lambda^{\prime}, c \epsilon_{m}\right)\right|} \int_{J\left(\lambda^{\prime}, c \epsilon_{m}\right)} \log \frac{1}{\rho\left(z, a_{j}\right)}|d z| \\
& \simeq \frac{1}{\left(1-r_{m}\right) \epsilon_{m}} \int_{J\left(\lambda^{\prime}, c \epsilon_{m}\right)} \log \frac{1}{\rho\left(z, a_{j}\right)}|d z|,
\end{aligned}
$$

where $J\left(\lambda^{\prime}, c \epsilon_{m}\right)=\left\{z:|z|=r_{m}\right\} \cap D\left(\lambda^{\prime}, c \epsilon_{m}\right)$. Then, for any $a_{1}, \ldots, a_{N} \in \tilde{Q}$,

$$
\begin{aligned}
\sum_{\lambda^{\prime} \in \Lambda_{Q}^{\prime}} \sum_{j=1}^{N} \log \frac{1}{\rho\left(\lambda^{\prime}, a_{j}\right)} & =\sum_{j=1}^{N} \sum_{\lambda^{\prime} \in \Lambda_{Q}^{\prime}} \log \frac{1}{\rho\left(\lambda^{\prime}, a_{j}\right)} \\
& \preceq \sum_{j=1}^{N} \frac{1}{\left(1-r_{m}\right) \epsilon_{m}} \int_{Q \cap\left\{|z|=r_{m}\right\}} \log \frac{1}{\rho\left(z, a_{j}\right)}|d z| .
\end{aligned}
$$

Since the length of $Q \cap\left\{|z|=r_{m}\right\}$ is approximately $2^{-n} \simeq 1-r_{m}$, we have, for some $r^{\prime}<1$,

$$
\frac{1}{1-r_{m}} \int_{Q \cap\left\{|z|=r_{m}\right\}} \log \frac{1}{\rho\left(z, a_{j}\right)}|d z| \preceq \int_{-r^{\prime}}^{r^{\prime}} \log \frac{1}{|x|} d x \simeq 1 .
$$

Hence

$$
\sum_{\lambda^{\prime} \in \Lambda_{Q}^{\prime}} \sum_{j=1}^{N} \log \frac{1}{\rho\left(\lambda^{\prime}, a_{j}\right)} \preceq N / \epsilon_{m},
$$

and using that $\# \Lambda_{Q}^{\prime} \geq 1 /\left(2 \epsilon_{m}\right)$ we get the desired result. 
From here we proceed as in the proof of Theorem 4.1. Let $N_{0}$ denote the set of $n \in \mathbb{N}$ for which there is some $r_{m}$ with $2^{-(n+1)}<1-r_{m} \leq 2^{-n}$. As pointed out before, there is no loss of generality in assuming that there is just one such $r_{m}$. To simplify the notation we re-index $r_{m}$ and call it $r_{n}$ (i.e. $\left\{r_{m}\right\}_{m \in \mathbb{N}}$ is re-indexed as $\left\{r_{n}\right\}_{n \in N_{0}}$ ).

Given a Blaschke sequence $Z$ and $\varepsilon$ small enough, define $\mathcal{Q}$ as in (4.3). The previous lemma ensures (3.4) in Theorem 3.2. In order to see that (3.3) also holds it is enough to show (4.4). Let $L_{n}$ as in (4.5). Since $\#\left(\Lambda \cap Q_{n, k}\right) \simeq$ $1 / \epsilon_{n}$ for $n \in N_{0}$, we have now

$$
\sum_{k:(n, k) \in \mathcal{Q}} N_{n, k} \geq \varepsilon \sum_{k:(n, k) \in \mathcal{Q}} \#\left(\Lambda \cap Q_{n, k}\right) \geq \varepsilon C L_{n} / \epsilon_{n}
$$

hence the Blaschke condition implies $\sum_{n \in N_{0}}\left(1-r_{n}\right) L_{n} / \epsilon_{n}<\infty$. If (4.4) does not hold by (4.6) we have $\sum_{n \in N_{0}} 1 / L_{n}<\infty$, and

$$
\sum_{n \in N_{0}}\left(\frac{1-r_{n}}{\epsilon_{n}}\right)^{1 / 2} \leq\left(\sum_{n \in N_{0}} \frac{1}{L_{n}}\right)^{1 / 2}\left(\sum_{n \in N_{0}} \frac{1-r_{n}}{\epsilon_{n}} L_{n}\right)^{1 / 2}<\infty
$$

which contradicts the hypothesis.

\subsection{Uniformly dense disks}

In this section we consider a different kind of sampling sets. We begin with the sequences considered by Ortega-Cerdà and Seip in [8].

Definition. A sequence $\Lambda \subset \mathbb{D}$ is uniformly dense if

(i) $\Lambda$ is separated, i.e. $\inf _{\lambda \neq \lambda^{\prime}} \rho\left(\lambda, \lambda^{\prime}\right)>0$.

(ii) There exists $r<1$ such that $\mathbb{D}=\bigcup_{\lambda \in \Lambda} D(\lambda, r)$.

Notice that, in the terminology of Section 4.1, uniformly dense sequences correspond to 1-nets.

Let $\varphi$ be a non-decreasing continuous function, bounded by some constant less than 1 . Given $\Lambda$ uniformly dense define $r_{\lambda}=\varphi(1-|\lambda|), D_{\lambda}^{\varphi}=$ $D\left(\lambda, r_{\lambda}\right)$ and the unions of disks

$$
\Lambda(\varphi):=\bigcup_{\lambda \in \Lambda} D_{\lambda}^{\varphi}
$$

Theorem 4.5. The set $\Lambda(\varphi)$ is sampling for $\mathcal{N}$ if and only if

$$
\int_{0}^{1} \frac{d t}{t \log (1 / \varphi(t))}=\infty
$$


In Section 5 we will see that this condition actually characterizes determination sets for the space of subharmonic functions in the disk having the characteristic growth of the Nevanlinna class.

Remark 4. Condition (4.7) is equivalent to the fact that the harmonic measure of the exterior boundary $\partial \mathbb{D}$ of $\mathbb{D} \backslash \overline{\Lambda(\varphi)}$ is zero, see [8, Theorem 1]. Notice also that for any fixed $K>1$ condition (4.7) is equivalent to

$$
\sum_{n} \frac{1}{\log \left(1 / \varphi\left(K^{-n}\right)\right)}=\infty
$$

Remark 5. The above family of examples allows us to see that there is no general relationship between $A^{-\alpha}$-sampling sets and Nevanlinna sampling sets.

A set $\Lambda \subset \mathbb{D}$ is sampling for the space

$$
A^{-\alpha}=\left\{f \in \operatorname{Hol}(\mathbb{D}):\|f\|_{\alpha}:=\sup _{z \in \mathbb{D}}(1-|z|)^{\alpha}|f(z)|<\infty\right\} \quad \alpha>0,
$$

when there exists $C>0$ such that

$$
\|f\|_{\alpha} \leq C \sup _{\lambda \in \Lambda}(1-|\lambda|)^{\alpha}|f(\lambda)|
$$

for all $f \in A^{-\alpha}$.

A well-known result of K. Seip [10, Theorem 1.1] characterizes $A^{-\alpha_{-}}$ sampling sets as those $\Lambda$ for which there exists a separated subsequence $\Lambda^{\prime} \subset \Lambda$ such that

$$
D_{-}\left(\Lambda^{\prime}\right):=\liminf _{r \rightarrow 1^{-}} \inf _{z \in \mathbb{D}} \frac{\sum_{\lambda: 1 / 2<\rho(\lambda, z)<r} \log \frac{1}{\rho(\lambda, z)}}{\log \frac{1}{1-r}}>\alpha .
$$

Let $\Lambda_{g}$ be a fine net associated to a function $g$ with $\int_{0} \frac{d t}{t^{1 / 2} g(t)}<\infty$, for instance $g(t)=t^{1 / 4}$. According to Theorem 4.1, $\Lambda_{g}$ is not a Nevanlinna sampling set. On the other hand, for any given $\alpha>0$, we can extract a maximal separated sequence $\Lambda^{\prime}$ with the separation small enough so that $D_{-}\left(\Lambda^{\prime}\right)>\alpha$, hence $\Lambda_{g}$ is $A^{-\alpha}$-sampling for all $\alpha>0$.

Also, given $\alpha>0$, consider a uniformly dense sequence $\Lambda$ with $D_{-}(\Lambda)<\alpha$ and take $\varphi$ satisfying $\lim _{t \rightarrow 0} \varphi(t)=0$ and (4.7). Then $\Lambda(\varphi)$ is Nevanlinna sampling but it is not $A^{-\alpha}$-sampling, since $D_{-}\left(\Lambda^{\prime}\right)<\alpha$ for any separated $\Lambda^{\prime} \subset \Lambda(\varphi)$.

Alternatively, take a set $\Lambda$ as in Section 4.2, sampling for $\mathcal{N}$, with $\lim _{n \rightarrow \infty} \frac{1-r_{n+1}}{1-r_{n}}=0$. Then $D_{-}(\Lambda)=0$, so it cannot be $A^{-\alpha}$-sampling for any $\alpha>0$. 
Proof. Assume that (4.7) does not hold. We will exhibit a function $f \in \mathcal{N}$ such that $\log |f(z)|=\log |B(z)|+\delta P_{z}(1)$ is bounded on $\Lambda(\varphi)$, for an appropriate choice of the Blaschke product $B$ and the constant $\delta>0$. Since, according to Lemma 2.5, $f \notin \mathcal{H}^{\infty}$, this will contradict the fact that $\Lambda(\varphi)$ is sampling.

Let $Z$ be the set of $\lambda \in \Lambda$ such that $m_{\lambda}:=\left[\frac{P_{\lambda}(1)}{\log 1 / \varphi(1-|\lambda|)}\right] \geq 1$, where each point $\lambda$ is taken with multiplicity $m_{\lambda}$.

In order to see that the Blaschke sum of $Z$ (with multiplicities) is finite, split it into the different dyadic squares $Q_{n, k}$. Notice that for $\lambda \in Q_{n, k}$

$$
P_{\lambda}(1)=\frac{1-|\lambda|^{2}}{|1-\lambda|^{2}} \simeq \frac{2^{-n}}{\left(2^{-n}+k 2^{-n}\right)^{2}}=\frac{2^{n}}{(1+k)^{2}} .
$$

Also, the uniform density condition implies $\# Z \cap Q_{n, k} \leq \# \Lambda \cap Q_{n, k} \preceq 1$. Therefore

$$
\begin{aligned}
\sum_{a \in Z}(1-|a|) & \simeq \sum_{n \geq 0} \sum_{k=0}^{2^{n}-1} \sum_{\lambda \in Z \cap Q_{n, k}} m_{\lambda}(1-|\lambda|) \\
& \simeq \sum_{n \geq 0} 2^{-n} \sum_{k=0}^{2^{n}-1} \sum_{\lambda \in Z \cap Q_{n, k}} \frac{P_{\lambda}(1)}{\log (1 / \varphi(1-|\lambda|))} \\
& \preceq \sum_{n \geq 0} \frac{1}{\log \left(1 / \varphi\left(2^{-n}\right)\right)}\left(\sum_{k=0}^{2^{n}-1} \frac{1}{1+k^{2}}\right) \simeq \int_{0}^{1} \frac{d t}{t \log (1 / \varphi(t))}<\infty
\end{aligned}
$$

On the other hand, if $z \in D_{\lambda}^{\varphi}$ we have

$$
\log |B(z)| \leq \log [\rho(z, \lambda)]^{m_{\lambda}} \preceq \frac{P_{\lambda}(1)}{\log 1 / \varphi(1-|\lambda|)} \log \varphi(1-|\lambda|)=-P_{\lambda}(1) .
$$

Therefore $\log |f|$ is bounded on $\bigcup_{\lambda \in \Lambda} D_{\lambda}^{\varphi}$ if $\delta$ is chosen small enough.

Assume now that (4.7) holds. By the uniform density condition, there exists $K>1$ such that for some $C>0$

$$
1 \leq \#\left\{\lambda: D_{\lambda}^{\varphi} \cap D(z, 1-1 / K) \neq \emptyset\right\} \leq C \quad \text { for all } z \in \mathbb{D} .
$$

There is no restriction in assuming that $K=2$, and equivalently, that

$$
1 \leq \#\left\{\lambda: D_{\lambda}^{\varphi} \cap Q_{n, k} \neq \emptyset\right\} \leq C \quad \text { for all } n \in \mathbb{N} \text { and } k=0, \ldots, 2^{n}-1 .
$$

In order to check the conditions of Theorem 3.2(a), and given a Blaschke sequence $Z$, let us see first that

$$
w_{n, k}\left(\Lambda(\varphi), N_{n, k}\right) \simeq N_{n, k} \log \frac{1}{\varphi\left(2^{-n}\right)} .
$$


Let $\lambda$ be such that $D_{\lambda}^{\varphi} \cap Q_{n, k} \neq \emptyset$. Take $a_{1}, \ldots, a_{N} \in D_{\lambda}^{\varphi}$; then $\rho\left(a_{j}, \lambda\right) \leq$ $\varphi\left(2^{-n}\right)$ and therefore, if there is only one such disk overlapping with $Q_{n, k}$,

$$
w_{n, k}\left(\Lambda(\varphi), N_{n, k}\right) \geq \sum_{j=1}^{N_{n, k}} \log \frac{1}{\rho\left(z, a_{j}\right)} \succeq N_{n, k} \log \frac{1}{\varphi\left(2^{-n}\right)} .
$$

If there is a finite number $C$ of such disks, put $N_{n, k} / C$ points in each disk, and the same result will hold.

On the other hand

$$
\begin{aligned}
\frac{1}{\left|D_{\lambda}^{\varphi}\right|} \int_{D_{\lambda}^{\varphi}} \sum_{j=1}^{N_{n, k}} \log \frac{1}{\rho\left(z, a_{j}\right)} d m(z) & \simeq \sum_{j=1}^{N_{n, k}} \frac{1}{\left(2^{-n} \varphi\left(2^{-n}\right)\right)^{2}} \int_{D_{\lambda}^{\varphi}} \log \frac{1}{\rho\left(z, a_{j}\right)} d m(z) \\
& \preceq \sum_{j=1}^{N_{n, k}} \frac{1}{\left(\varphi\left(2^{-n}\right)\right)^{2}} \int_{D\left(0, \varphi\left(2^{-n}\right)\right)} \log \frac{1}{|z|} d m(z) \\
& =\sum_{j=1}^{N_{n, k}} \log \frac{1}{\varphi\left(2^{-n}\right)}=N_{n, k} \log \frac{1}{\varphi\left(2^{-n}\right)}
\end{aligned}
$$

which proves the reverse estimate.

$$
\text { Let } N_{n}=\sum_{k=0}^{2^{n}-1} N_{n, k}, \gamma_{n} \in(0,1) \text { to be determined later, and } L_{n}=\left[\left(1-\gamma_{n}\right) N_{n}\right] \text {. }
$$

Fixed $n \in \mathbb{N}$, let $\mathcal{Q}_{n}^{c}$ be the set of indices $(n, k)$ corresponding to the $L_{n}$ dyadic squares $Q_{n, k}$ with the largest values of $N_{n, k}$. By definition

$$
\sum_{(n, k) \in \mathcal{Q}_{n}^{c}} N_{n, k} \geq L_{n}
$$

Call $\mathcal{Q}_{n}$ the remaining indices $(n, k)$ and define $\mathcal{Q}=\cup_{n} \mathcal{Q}_{n}$. Then

$$
\sum_{(n, k) \in \mathcal{Q}} 2^{-n} w_{n, k}\left(\Lambda(\varphi), N_{n, k}\right) \simeq \sum_{n} 2^{-n}\left(\log \frac{1}{\varphi\left(2^{-n}\right)}\right)\left(\sum_{(n, k) \in \mathcal{Q}_{n}} N_{n, k}\right) .
$$

Since

$$
\sum_{(n, k) \in \mathcal{Q}_{n}} N_{n, k}=N_{n}-\sum_{(n, k) \in \mathcal{Q}_{n}^{c}} N_{n, k} \leq N_{n}-L_{n} \leq N_{n} \gamma_{n},
$$

condition (3.4) is now equivalent to

$$
\sum_{n} \gamma_{n}\left(\log \frac{1}{\varphi\left(2^{-n}\right)}\right) 2^{-n} N_{n}<\infty
$$

Condition (4.8) implies

$$
\liminf _{n \rightarrow \infty}\left(\log \frac{1}{\varphi\left(2^{-n}\right)}\right) 2^{-n} N_{n}=0
$$


since otherwise $2^{-n} N_{n} \succeq\left(\log 1 / \varphi\left(2^{-n}\right)\right)^{-1}$ and the Blaschke condition would be violated. In particular, there exists a subsequence such that

$$
\sum_{j}\left(\log \frac{1}{\varphi\left(2^{-n_{j}}\right)}\right) 2^{-n_{j}} N_{n_{j}}<\infty
$$

Define

$$
\gamma_{n}= \begin{cases}1-1 / N_{n} & \text { if } n=n_{j} \\ \left(\log 1 / \varphi\left(2^{-n}\right)\right)^{-1} & \text { if } n \neq n_{j}\end{cases}
$$

Then (3.4) holds:

$$
\sum_{n} \gamma_{n}\left(\log \frac{1}{\varphi\left(2^{-n}\right)}\right) 2^{-n} N_{n} \leq \sum_{j}\left(\log \frac{1}{\varphi\left(2^{-n_{j}}\right)}\right) 2^{-n_{j}} N_{n_{j}}+\sum_{n} 2^{-n} N_{n}<\infty .
$$

To prove (3.3) in Theorem 3.2 we use an argument as in (4.6). Here

$$
\sum_{n=0}^{\infty} \sum_{(n, k) \in \mathcal{Q}_{n}^{c}}\left(\frac{2^{-n}}{2^{-n}+k 2^{-n}}\right)^{2} \geq \sum_{n} \frac{1}{L_{n}} \geq \sum_{n} \frac{1}{\left(1-\gamma_{n}\right) N_{n}} \geq \sum_{j} 1=\infty
$$

as desired.

\section{Uniformly dense disks for subharmonic functions}

In this section we show that Theorem 4.5, with a different proof, can be extended to the class

$$
\mathcal{S N}=\left\{u: \mathbb{D} \longrightarrow \mathbb{R} \text { subharmonic with } \sup _{r<1} \int_{0}^{2 \pi} u^{+}\left(r e^{i \theta}\right) d \theta<\infty\right\} .
$$

A set $\Lambda \subset \mathbb{D}$ is called a determination set for $\mathcal{S N}$ if $\sup _{\Lambda} u=\sup _{\mathbb{D}} u$ for all $u \in \mathcal{S N}$.

Theorem 5.1. A uniformly dense family of disks $\Lambda(\varphi)$ is a determination set for $\mathcal{S N}$ if and only if (4.7) holds.

Proof. The necessity of (4.7) is contained in Theorem 4.5, since log $|f| \in$ $\mathcal{S N}$ whenever $f \in \mathcal{N}$.

Assume now that (4.7) holds. Let $u \in \mathcal{S N}$ be such that $\sup _{\Lambda(\varphi)} u \leq 0$. We want to prove that $u(p) \leq 0$ for all $p \notin \Lambda(\varphi)$.

Let $R_{n}=1-K^{-n}$, where $K>1$ will be chosen later on, and consider the domains

$$
\Omega_{n}(p, \Lambda, \varphi)=D\left(p, R_{n}\right) \backslash \bigcup_{\lambda: D_{\lambda}^{\varphi} \subset D\left(p, R_{n}\right)} \overline{D_{\lambda}^{\varphi}}
$$


Let $\omega(A ; p, \Omega)$ denote the harmonic measure at $p$ of a set $A \subset \partial \Omega$, and let $\phi_{p}$ denote the automorphism of $\mathbb{D}$ exchanging $p$ and 0 . The subharmonicity of $u^{+}$gives then

$$
\begin{aligned}
u^{+}(p) & \leq \int_{\partial \Omega_{n}(p, \Lambda, \varphi)} u^{+}(\zeta) d \omega\left(\zeta ; p, \Omega_{n}(p, \Lambda, \varphi)\right) \\
& =\int_{\partial D\left(p, R_{n}\right)} u^{+}(\zeta) d \omega\left(\zeta ; p, \Omega_{n}(p, \Lambda, \varphi)\right) \\
& =\int_{|\zeta|=R_{n}}\left(u^{+} \circ \phi_{p}\right)(\zeta) d \omega\left(\zeta ; 0, \phi_{p}\left(\Omega_{n}(p, \Lambda, \varphi)\right)\right) .
\end{aligned}
$$

First observe that the harmonic measure in $\phi_{p}\left(\Omega_{n}(p, \Lambda, \varphi)\right)$ can be estimated by the harmonic measure of a domain $\Omega_{n}(0, \tilde{\Lambda}, \psi)$, where $\tilde{\Lambda}$ is uniformly dense and $\psi$ is a non-decreasing, continuous function bounded by some constant less than 1 satisfying (4.7). To see this let $\tilde{\Lambda}=\phi_{p}(\Lambda)$, consider the hyperbolic rings

$$
A_{n}=\left\{z \in \mathbb{D}: R_{n-1} \leq \rho(z, p)<R_{n}\right\}
$$

and take $\psi$ non-decreasing, continuous, and such that $\psi\left(R_{n}\right)=\min _{A_{n}} \varphi$. Then $D_{\phi_{p}(\lambda)}^{\psi} \subset D_{\phi_{p}(\lambda)}^{\varphi}$ and therefore

$$
\omega\left(A ; 0, \phi_{p}\left(\Omega_{n}(p, \Lambda, \varphi)\right)\right) \leq \omega\left(A ; 0, \Omega_{n}(0, \tilde{\Lambda}, \psi)\right)
$$

for any $A \subset\left\{|z|=R_{n}\right\}$. Notice also that $\min _{A_{n}} \varphi$ is attained for $z$ with

$$
1-|z|=\frac{\left(1-R_{n}\right)(1-|p|)}{1+R_{n}|p|} \leq\left(1-R_{n}\right)(1-|p|)=K^{-n}(1-|p|),
$$

and therefore

$$
\begin{aligned}
\int_{0}^{1} \frac{d t}{t \log (1 / \psi(t))} & \simeq \sum_{n=1}^{\infty} \frac{1}{\log \left(1 / \psi\left(K^{-n}\right)\right)} \leq \sum_{n=1}^{\infty} \frac{1}{\log \left(1 / \varphi\left(K^{-n}(1-|p|)\right)\right.} \\
& \simeq \int_{0}^{1} \frac{d t}{t \log (1 / \varphi(t))} .
\end{aligned}
$$

We have thus

$$
u^{+}(p) \leq \int_{|\zeta|=R_{n}}\left(u^{+} \circ \phi_{p}\right)(\zeta) d \omega\left(\zeta ; 0, \Omega_{n}(0, \tilde{\Lambda}, \psi)\right) .
$$

As mentioned in Remark 4, the hypothesis implies $\omega(\partial \mathbb{D} ; 0, \mathbb{D} \backslash \overline{\tilde{\Lambda}(\psi)})=0$. In order to see that the previous integrals tend to zero we need a slight refinement of Theorem 1 in [8]. Let $d \sigma_{n}=d \theta /\left(2 \pi R_{n}\right)$ denote the normalized Lebesgue measure in $|z|=R_{n}$. 
Lemma 5.2. Given a uniformly dense sequence $\Lambda$ and a non-decreasing continuous function $\varphi$ satisfying (4.7), there exist $R_{n}<1$ with $\lim _{n} R_{n}=1$, and $\epsilon_{n}>0$ with $\lim _{n} \epsilon_{n}=0$ such that $\omega\left(I ; 0, \Omega_{n}(0, \Lambda, \varphi)\right) \leq \epsilon_{n} \sigma_{n}(I)$ for all intervals $I \subset\left\{\zeta:|\zeta|=R_{n}\right\}$.

Once this lemma is proved, the above estimate yields

$$
u^{+}(p) \leq \epsilon_{n} \int_{|\zeta|=R_{n}}\left(u^{+} \circ \phi_{p}\right)(\zeta) d \sigma_{n}(\zeta) \leq \frac{\epsilon_{n}}{R_{n}} \sup _{r<1} \int_{0}^{2 \pi}\left(u^{+} \circ \phi_{p}\right)\left(r e^{i \theta}\right) \frac{d \theta}{2 \pi},
$$

and letting $n \rightarrow \infty$ we obtain $u^{+}(p) \leq 0$, as desired.

Proof of Lemma 5.2. We prove this by induction. We drop the superindex in $D_{\lambda}^{\varphi}$ and denote $\Omega_{n}(0, \Lambda, \varphi)$ simply by $\Omega_{n}$.

There is no restriction in assuming that there are no $D_{\lambda}$ in $D\left(0, R_{1}\right)$; thus $\omega\left(I ; 0, \Omega_{1}\right) \leq|I|$ for all intervals $I \subset\left\{\zeta:|\zeta|=R_{1}\right\}$.

We have

$$
\omega\left(I ; 0, \Omega_{n}\right)=\int_{|z|=R_{n-1}} P(z \rightarrow I) d \omega\left(z ; 0, \Omega_{n-1}\right),
$$

where $P(z \rightarrow I)$ denotes the probability that a Brownian motion starting at $z$ exits $\Omega_{n}$ through $I$. The hypothesis of induction gives

$$
\omega\left(I ; 0, \Omega_{n}\right) \leq \epsilon_{n-1} \int_{|z|=R_{n-1}} P(z \rightarrow I) d \sigma_{n-1}(z) .
$$

In the estimate of $P(z \rightarrow I)$ we use the uniform density of $\Lambda$ : there exist $\delta \in(0,1)$ and $K>1$ (independent of $n$ ) such that for each $z \in\left\{|z|=R_{n-1}\right\}$ there is $\lambda \in \Lambda$ with $D_{\lambda} \in D\left(0, R_{n}\right) \backslash D\left(0, R_{n-1}\right)$ and $\rho(z, \lambda) \leq \delta$. Then

$$
P(z \rightarrow I) \leq \omega\left(I ; z, D\left(0, R_{n}\right) \backslash D_{\lambda}\right) .
$$

This harmonic measure can be estimaded by comparing with an explicit harmonic function. Let $\Psi_{n}(z)=z / R_{n}$, which sends $D\left(0, R_{n}\right)$ to $\mathbb{D}$, and let $P_{z}^{(n)}(\zeta)$ denote the Poisson kernel in $D\left(0, R_{n}\right)$. Let $\lambda^{(n)} \in \mathbb{D}, r_{\lambda}^{(n)} \in(0,1)$ be such that $\Psi_{n}\left(D_{\lambda}\right)=\Psi_{n}\left(D\left(\lambda, r_{\lambda}\right)\right)=D\left(\lambda^{(n)}, r_{\lambda}^{(n)}\right)$, and define the harmonic function on $D\left(0, R_{n}\right) \backslash D_{\lambda}$

$$
F_{\lambda}(z, I)=\omega\left(I ; z, D\left(0, R_{n}\right)\right)-\left(\inf _{w \in \partial D_{\lambda}} \omega\left(I ; w, D\left(0, R_{n}\right)\right)\right) \frac{\log \rho\left(\lambda^{(n)}, \Psi_{n}(z)\right)}{\log r_{\lambda}^{(n)}}
$$

It is clear that $\omega\left(I ; \eta, D\left(0, R_{n}\right) \backslash D_{\lambda}\right) \leq F_{\lambda}(\eta, I)$ for $\eta$ in the boundary of $D\left(0, R_{n}\right) \backslash D_{\lambda}$, and therefore in all $D\left(0, R_{n}\right) \backslash D_{\lambda}$. Hence

$$
P(z \rightarrow I) \leq F_{\lambda}(z, I) \text {. }
$$


We want to give an estimate of $F_{\lambda}(z, I)$ which does not depend on $\lambda$. Using that $\omega\left(I ; z, D\left(0, R_{n}\right)\right)=\int_{I} P_{z}^{(n)}(\zeta) d \sigma_{n}(\zeta)$, we can write

$$
\begin{aligned}
F_{\lambda}(z, I)=\left(\int_{I} P_{z}^{(n)}(\zeta) d \sigma_{n}(\zeta)\right) \times & \\
& \times\left(1-\frac{\left.\inf _{w \in \partial D_{\lambda} \int_{I} P_{w}^{(n)}(\zeta) d \sigma_{n}(\zeta)} \frac{\log \rho\left(\lambda^{(n)}, \Psi_{n}(z)\right)}{\int_{I} P_{z}^{(n)}(\zeta) d \sigma_{n}(\zeta)}\right) .}{\log r_{\lambda}^{(n)}} .\right.
\end{aligned}
$$

Since $\lim _{|\lambda| \rightarrow 1} r_{\lambda}=0$ and $\rho(z, \lambda) \leq \delta$, by the Harnack's estimates (or by a direct computation), there exists $c>0$ such that

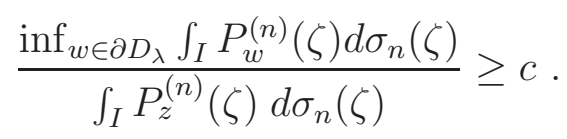

Also, there exists $\delta^{\prime}>0$ such that for $n$ big enough $\rho\left(\lambda^{(n)}, \Psi_{n}(z)\right) \leq \delta^{\prime}$. With this and the fact that $K^{-n-1} \leq 1-|\lambda|<K^{-n}$ we deduce that there exists some $C>0$ such that

$$
P(z \rightarrow I) \leq\left(\int_{I} P_{z}^{(n)}(\zeta) d \sigma_{n}(\zeta)\right)\left(1-\frac{C}{\log 1 / \varphi\left(K^{-n}\right)}\right)
$$

From (5.1) we have therefore

$$
\begin{aligned}
\omega\left(I ; 0, \Omega_{n}\right) & \leq \epsilon_{n-1}\left(1-\frac{C}{\log 1 / \varphi\left(K^{-n}\right)}\right) \int_{I} \int_{|z|=R_{n-1}} P_{z}^{(n)}(\zeta) d \sigma_{n-1}(z) d \sigma_{n}(\zeta) \\
& =\epsilon_{n-1}\left(1-\frac{C}{\log 1 / \varphi\left(K^{-n}\right)}\right) \sigma_{n}(I) .
\end{aligned}
$$

Defining

$$
\epsilon_{n}=\epsilon_{n-1}\left(1-\frac{C}{\log 1 / \varphi\left(K^{-n}\right)}\right)=\prod_{j=1}^{n}\left(1-\frac{C}{\log 1 / \varphi\left(K^{-j}\right)}\right)
$$

and using Remark 4 we obtain the stated properties.

\section{Appendix}

Here we give a direct proof that determination sets for $\mathcal{N}$ are Hayman-Lyons sets. According to [3, Corollary 2] this implies $|N T(\Lambda)|=2 \pi$, and therefore $\Lambda$ is determination set for $\mathcal{H}^{\infty}$.

Let $\delta \in(0,1)$ and let $\Lambda_{0} \subset \Lambda$ be maximal among the subsequences of $\Lambda$ such that $\rho\left(\lambda, \lambda^{\prime}\right) \geq \delta$ for all $\lambda, \lambda^{\prime} \in \Lambda_{0}, \lambda \neq \lambda^{\prime}$. We want to prove that

$$
\sum_{\lambda \in \Lambda_{0}}(1-|\lambda|) P_{\lambda}(\zeta)=\infty \quad \text { for all } \zeta \in \mathbb{T} .
$$


There is no loss of generality in reducing ourselves to the case $\zeta=1$. Also, we restrict our attention to those $\lambda \in \Lambda_{0}$ with $P_{\lambda}(1) \geq 1$, and denote by $\tilde{\Lambda}_{0}$ the subsequence made with such points. Notice that $\Lambda \cap\left\{z: P_{z}(1) \leq 1\right\}$ cannot be a determination sequence for $\mathcal{N}$ anyway, as the function $f \in \mathcal{N}$ with $\log |f(z)|=P_{z}(1)$ shows. Thus, let us assume that

$$
\sum_{\lambda \in \tilde{\Lambda}_{0}}(1-|\lambda|) P_{\lambda}(1)<\infty
$$

and see that there exists $f \in \mathcal{N} \backslash \mathcal{H}^{\infty}$ with $\sup _{\Lambda}|f|<\infty$.

Consider the sequence $Z$ consisting of the points $\lambda \in \tilde{\Lambda}_{0}$, with multiplicity $\left[P_{\lambda}(1)\right]$. By assumption $Z$ is a Blaschke sequence, and therefore, for any $C>0$, there exists $f \in \mathcal{N}$ such that

$$
\log |f(z)|=\log \left|B_{Z}(z)\right|+C P_{z}(1) .
$$

As seen in Example 1, such $f$ cannot be bounded in the whole disk.

We want to choose $C$ so that $\sup _{\Lambda}|f|<\infty$. By construction, we only need to consider $\lambda \notin \tilde{\Lambda}_{0}$. We separate two cases:

(i) If $P_{\lambda}(1) \leq 2$ obviously $\log |f(\lambda)| \leq 2 C$.

(ii) If $P_{\lambda}(1)>2$ there exists $\lambda_{0} \in \tilde{\Lambda}_{0}$ such that $\rho\left(\lambda, \lambda_{0}\right) \leq \delta$. By Harnack's inequalities we obtain:

$$
\begin{aligned}
\log |f(\lambda)| & \leq \log \rho\left(\lambda, \lambda_{0}\right)^{\left[P_{\lambda_{0}}(1)\right]}+C P_{\lambda}(1) \\
& \leq \frac{1}{2}(\log \delta) P_{\lambda_{0}}(1)+C\left(\frac{1+\delta}{1-\delta}\right) P_{\lambda_{0}}(1) .
\end{aligned}
$$

Choosing $C=\frac{1}{2} \frac{1-\delta}{1+\delta} \log \frac{1}{\delta}$ we see that in this case $\log |f(\lambda)| \leq 0$, as desired.

\section{References}

[1] Beurling, A.: A minimum principle for positive harmonic functions. Ann. Acad. Sci. Fenn. A I 372 (1965), 7 pp.

[2] Brown, L., Shields, A. ANd Zeller, K.: On absolutely convergent exponential sums. Trans. Amer. Math. Soc. 96 (1960), 162-183.

[3] Gardiner, S. J.: Sets of determination for harmonic functions. Trans. Amer. Math. Soc. 338 (1993), no. 1, 233-243.

[4] Garnett, J. B.: Bounded analytic functions. Pure and Applied Mathematics 96. Academic Press, New York-London, 1981.

[5] Hayman, W. K. and Lyons, T. J.: Bases for positive continuous functions. J. London Math. Soc. (2) 42 (1990), 292-308. 
[6] Hartmann, A., Massaneda, X., Nicolau, A., and Thomas, P. J.: Interpolation in the Nevanlinna and Smirnov classes and harmonic majorants. J. Funct. Anal. 217 (2004), 1-37.

[7] Nikolski, N.K.: Operators, functions, and systems: an easy reading. Vol. 1. Hardy, Hankel, and Toeplitz. Vol. 2. Model operators and systems. Mathematical Surveys and Monographs 92 and 93. American Mathematical Society, Providence, RI, 2002.

[8] Ortega-Cerdì, J. And Seip, K.: Harmonic measure and uniform densities. Indiana Univ. Math. J. 53 (2004), no. 3, 905-923.

[9] Rosenblum, M. and Rovnyak, J.: Hardy classes and operator theory. Oxford Mathematical Monographs. Oxford Science Publications. The Clarendon Press, Oxford University Press, New York, 1985.

[10] SEIP, K.: Beurling type density theorems in the unit disk. Invent. Math. 113 (1993), 21-39.

[11] Shapiro, J. H. And Shields, A. L.: Unusual topological properties of the Nevanlinna class. Amer. J. Math. 97 (1975), no. 4, 915-936.

[12] Thomas, P. J.: Sampling sets for Hardy spaces of the disk. Proc. Amer. Math. Soc. 126 (1998), 2927-2932.

Recibido: 18 de octubre de 2006

Xavier Massaneda Departament de Matemàtica Aplicada i Anàlisi Universitat de Barcelona Gran Via 585

08007-Barcelona, Spain xavier.massaneda@ub.edu

Pascal J. Thomas Institut de Mathématiques de Toulouse CNRS UMR 5219 (UFR MIG) Université Paul Sabatier 31062 Toulouse Cedex 9, France pthomas@cict.fr

Both authors supported by a XTI program of the Comunitat de Treball dels Pirineus. First author also supported by the DGICYT grant MTM2005-08984-C02-02 and the CIRIT grant 2005-SGR00611. 